Document downloaded from:

http://hdl.handle.net/10251/154392

This paper must be cited as:

Corrales, AR.; Carrillo, L.; Lasierra, P.; Nebauer, SG.; Dominguez-Figueroa, J.; RenauMorata, B.; Pollmann, S.... (2017). Multifaceted role of cycling DOF factor 3 (CDF3) in the regulation of flowering time and abiotic stress responses in Arabidopsis. Plant Cell \& Environment. 40(5):748-764. https://doi.org/10.1111/pce.12894

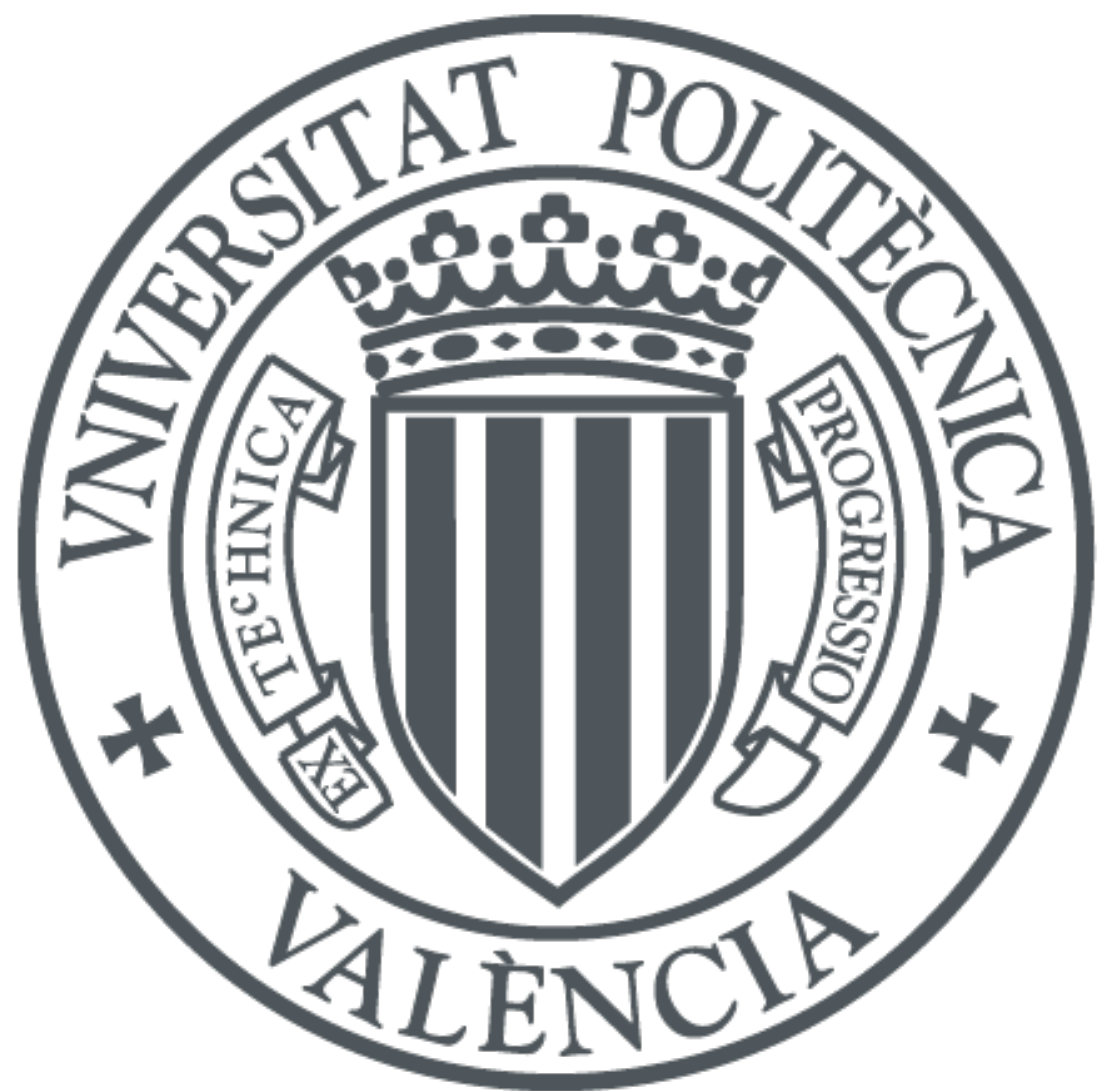

The final publication is available at https://doi.org/10.1111/pce.12894

Copyright Blackwell Publishing Additional Information 


\section{Title: Multifaceted Role of Cycling Dof Factor 3 (CDF3) in the regulation of flowering time and abiotic stress responses in Arabidopsis}

Alba-Rocio Corrales ${ }^{1+a}$, Laura Carrillo ${ }^{1+}$, Pilar Lasierra ${ }^{2+}$, Sergio G. Nebauer ${ }^{3}$, Jose Dominguez-Figueroa $^{1}$, Begoña Renau-Morata ${ }^{3}$, Stephan Pollmann ${ }^{1}$, Antonio Granell$^{4}$, Rosa-Victoria Molina ${ }^{3}$, Jesús Vicente-Carbajosa* ${ }^{* 1}$, Joaquín Medina*1.

1. Centro de Biotecnología y Genómica de Plantas. Universidad Politécnica de Madrid (UPM) - Instituto Nacional de Investigación y Tecnología Agraria y Alimentaria (INIA). Campus de Montegancedo. Autopista M40 (km 38). 28223 Madrid, Spain.

2. Centro Nacional de Biotecnología-CSIC, C/Darwin 3, 28049 Madrid, Spain.

3. Departamento de Producción Vegetal, Universitat Politècnica de València. Camino de Vera s/n, 46022 Valencia, Spain.

4. Instituto de Biología Molecular y Celular de Plantas (CSIC-UPV), Ingeniero Fausto Elio s/n, 46022 Valencia, Spain.

+. These authors contributed equally to this article.

a. Actual address: Universidad Santiago de Cali. Colombia.

*Correspondence should be addressed: jesus.vicente@upm.es; medina.joaquin@inia.es

Centro de Biotecnología y Genómica de Plantas (UPM-INIA). Campus 22 Montegancedo. Autopista M40 (km 38), 28223-Pozuelo de Alarcón, Madrid, 23 Spain.

Phone: 34-914524900

Fax: 34-917517721

Figures: 7

Supporting information includes: 8 Figures, 4 tables, 1 file.

This article has been accepted for publication and undergone full peer review but has not been through the copyediting, typesetting, pagination and proofreading process which may lead to differences between this version and the Version of Record. Please cite this article as doi: 10.1111/pce.12894 


\section{ABSTRACT}

DOF (DNA-binding with one finger)-type transcription factors are involved in many fundamental processes in higher plants, from responses to light and phytohormones to flowering time and seed maturation, but their relation with abiotic stress tolerance is largely unknown. Here, we identify the roles of CDF3, an Arabidopsis DOF gene in abiotic stress responses and developmental processes like flowering time. CDF3 is highly induced by drought, extreme temperatures and abscisic acid treatment. The CDF3 T-DNA insertion mutant cdf3-1 is much more sensitive to drought and low temperature stress, whereas CDF3 overexpression enhances the tolerance of transgenic plants to drought, cold and osmotic stress and promotes late flowering. Transcriptome analysis revealed that CDF3 regulates a set of genes involved in cellular osmoprotection and oxidative stress, including the stress tolerance transcription factors CBFs, DREB2A, and ZAT12, which involve both GIGANTEAdependent and independent pathways. Consistently, metabolite profiling disclosed that the total amount of some protective metabolites including GABA, proline, glutamine and sucrose were higher in CDF3-overexpressing plants. Taken together, these results indicate that CDF3 plays a multifaceted role acting on both flowering time and abiotic stress tolerance, in part by controlling the CBF/DREB2A-CRT/DRE and ZAT10/12 modules.

\section{Short Abstract}

The present study provides new notions about the function of DOF Transcription factors and unveils CDF3 as a key factor that display multiple roles related to plant responses to adverse environmental conditions and the developmental program underlying the transition from vegetative to reproductive phase.

Key words: Drought stress, low temperature stress, nitrogen, flowering time, DOF, CDF, gene expression, Arabidopsis. 


\section{INTRODUCTION}

Abiotic stresses such as drought and extreme temperatures are among the most important environmental factors that limit plant growth, development and productivity. Plants have developed sophisticated molecular, biochemical and physiological mechanisms to adjust growth according to the availability of resources and to environmental conditions (Xiong et al., 2002; Zhu, 2002; Shinozaki and YamaguchiShinozaki, 2007; Ahuja et al., 2010; Skirycz and Inze 2010; Osakabe et al., 2011; Nishiyama et al., 2012). Transcriptome analyses have identified a number of genes that are inducible by abiotic stresses (Seki et al., 2002; Shinozaki et al., 2003; Yamaguchi-Shinozaki and Shinozaki, 2006) encoding for proteins with function in stress tolerance, including osmoregulatory and antioxidant proteins, chaperones, detoxification enzymes and LEA (LLate Embryogenesis Abundant) proteins (Yamaguchi-Shinozaki and Shinozaki, 2004; 2006; Gong et al., 2010) and genes involved in signal transduction and the control of gene expression, such as protein kinases, protein phosphatases, enzymes involved in phospholipid metabolism (Seki et al., 2003; Shinozaki and Yamaguchi-Shinozaki, 2006) and various transcription factors (TFs). These stress-inducible transcription factors are members of the DREB, ERF, WRKY, MYB, bHLH, bZIP, DOF and NAC families (Shinozaki et al., 2003).

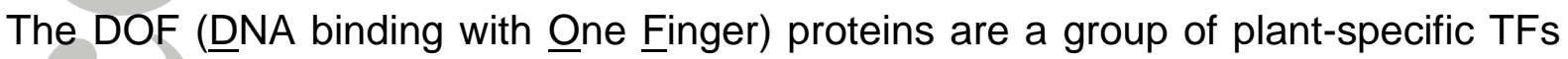
that contain a DNA-binding domain usually located close to the $\mathrm{N}$-terminal region of the protein. This DOF domain is a highly conserved region of 52 amino acid residues with a $\mathrm{C}_{2}-\mathrm{C}_{2}$ finger structure associated to a basic region that binds specifically to cis regulatory elements containing the common core 5'-T/AAAG-3' motif (Yanagisawa and Schmidt, 1999; Noguero et al., 2013). In contrast, the C-terminal end is a highly variable region that contains the transcriptional regulatory elements and it might 
reflect diverse functions of different DOF proteins (Yanagisawa 2001; Hong-Feng et al., 2013).

In previous studies, DOF proteins have been reported to be involved in the regulation of a variety of biological processes including seed maturation, germination and hormone signaling (Reviewed by Yanagisawa 2002; Noguero et al., 2013). Moreover, DOF TFs such as maize DOF1 and DOF2 have also been involved in the control of carbon and nitrogen metabolism through the regulation of phosphoenolpyruvate carboxykinase (PECPK), glutamine synthase (GS) and glutamate synthase (GLU) (Yanagisawa and Sheen 1998; Yanagisawa et al., 2004; Rueda-Lopez et al., 2008; Kurai et al., 2011).

Arabidopsis genome encodes 36 DOF TFs. Phylogenetic studies using the complete set of amino acid sequences of DOF proteins from Arabidopis, rice, tomato and Brachypodium, identified four major clusters of orthologous genes or subfamilies (A, B, C and D) (Lijavetzky et al., 2003; Hernando-Amado et al., 2012; Corrales et al., 2014a). The group D contains a cluster of DOF factors whose transcripts oscillate under constant light conditions and are known as Cycling Dof Factors (CDF1-5) (Imaizumi et al., 2005; Fornara et al., 2009). It is well established that CDF transcription factors display an important role in the photoperiodic flowering-time control in Arabidopsis, by modulating the diurnal expression rhythm of CONSTANS (CO) expression and consequently FT expression. In addition, the stability of CDF proteins is compromised under long days by a protein complex including FLAVINBINDING KELCH REPEAT F-BOX PROTEIN (FKF1) and GIGANTEA (GI) (Imaizumi et al., 2005; Sawa et al., 2007; Jarillo et al., 2008; Fornara et al., 2009). Recently, data reported by Corrales et al., (2014a) has shown that tomato CDFs (SICDFs) display additional functions as transcriptional regulators. The overexpression of 
tomato SICDF1 and SICDF3 in Arabidopsis enhanced drought and salt tolerance. Moreover, the overexpression in Arabidopsis of SICDF3 but not SICDF1 promotes late flowering by modulating the expression of $C O$ and $F T$, suggesting that CDFs might play a central role in abiotic stress tolerance, along with their role in flowering time control.

In this study, we find that in Arabidopsis CDF3 is particularly induced by drought, salt, extreme temperatures and $A B A$. We show that $C D F 3$ overexpression enhances plant tolerance to drought, cold and osmotic stress, whereas down-regulation of CDF3 (cdf3-KO) leads to attenuated resistance. Moreover we demonstrate that CDF3 regulates a set of genes involved in cellular osmoprotection and ROS homeostasis, which are associated with changes in sugar and amino acid levels in stressed plants through both GI- dependent and -independent pathways. These findings suggest that Arabidopsis CDF3 plays multiple roles in both abiotic stress responses besides its known role in flowering time signal transduction pathways.

\section{MATERIAL AND METHODS}

\section{Plant material and growth conditions}

The Arabidopsis thaliana ecotype Columbia (Col-0) was used as WT. The cdf3-1 TDNA insertion knockout mutant was obtained from the GABI-Kat collection (GK808605; Rosso et al., 2003). Seeds were surface-sterilized and stratification was performed by planting seeds on half strength MS (MS/2) medium (Murashige and Skoog, 1962) containing $0.5 \%(\mathrm{w} / \mathrm{v})$ sucrose and $0.8 \%(\mathrm{w} / \mathrm{v})$ agar and incubating them at $4{ }^{\circ} \mathrm{C}$ for 2 days. After germination, 10-day-old seedlings were transferred to soil and grown in a growth chamber at $22{ }^{\circ} \mathrm{C}$ under LD (16/8 h light/dark) conditions. 


\section{Plasmid constructs and Arabidopsis transformation}

The open reading frame (ORF) of CDF3 gene was cloned into a binary vector pGWB2 under the control of the 35S CaMV promoter (Karimi et al., 2007). The resultant plasmid was used to transform Arabidopsis thaliana (Col-0) plants by the floral dip method (Clough and Bent, 1998). For $\beta$-GLUCURONIDASE (GUS) histochemical staining experiments the promoter regions of the CDF3 and CRUCIFERIN (CRU) genes (from -1060bp and $-1200 \mathrm{bp}$ to the ATG, respectively) were cloned into a binary vector containing a GUS reporter gene (uidA), producing an in-phase fusion with the reporter gene constructs $p C D F 3:: G U S$ and $p C R U:: G U S$, respectively. The corresponding plasmids were used to transform Arabidopsis plants.

\section{Subcellular localization of CDF3 and histochemical GUS staining}

For epifluorescence and light microscopy, 10-day-old Arabidopsis seedlings and onion epidermal cells were analyzed with a Confocal Laser Scanning microscope as described previously (Corrales et al., 2014a). For GUS staining pCDF3::GUS and pCRU::GUS transgenic plants were used as described by Jefferson et al, (1987). See Appendix S1 for details.

\section{Protoplast transformation}

Mesophyll protoplasts were isolated from rosette leaves of 4-week-old-Arabidopsis plants ecotype Columbia (Col-0) grown in soil $\left(21 / 18{ }^{\circ} \mathrm{C}, 8 / 16 \mathrm{~h}\right.$ light/dark). Protoplast isolation and transfection was performed according to the method described previously (Alonso et al., 2009; Yoo et al., 2007). For more details see Appendix S1. 


\section{RNA isolation, RT-PCR and qRT-PCR}

Total RNA was extracted following Oñate-Sanchez and Vicente-Carbajosa, (2008). RT-PCR, and qRT-PCR analyses were carried out as described previously (Corrales et al., 2014a,b; Catala et al., 2011). For more detail please see Appendix S1.

\section{Germination and post-germinative growth assay}

Germination analyses and assays on post-germinative behaviour and root growth were performed as described previously (Corrales et al., 2014b; Appendix S1). The assays were carried out using Col-0, cdf3-1 and 35S::CDF3 lines. Seeds were collected at the same time and obtained from plants grown under the same conditions.

\section{Photosynthesis and leaf fluorescence measurement}

Net photosynthesis and related gas exchange variables, stomatal conductance and substomatal $\mathrm{CO}_{2}$ were determined using an LI-6400 infrared gas analyser (LICOR Biosciences, Lincoln, USA) as described previously by (Galmes et al., 2007). Measurements were performed at steady state under saturating light (PAR 1000 $\left.\mu \mathrm{mol} \mathrm{m} \mathrm{m}^{-2}\right), 400 \mathrm{ppm} \mathrm{CO}_{2}$, ambient temperature and a vapour pressure difference (vpd) between 1 and $2 \mathrm{kPa}$. Maximum photochemical efficiency (Fv/Fm) on darkadapted leaves was measured using a portable pulse amplitude modulation fluorometer (MINI PAM, Walz, Effeltrich Germany). Responses to osmotic stress were performed using 3-week-old cdf3-1, 35S::CDF3 and Col-0 plants that were transplanted to hydroponic culture, and photosynthesis parameters were measured after 7 days of growth by adding 5\% PEG-8000 (24h). 


\section{Drought and cold stress tolerance assay}

Drought and cold stress assays were carried out as described previously (Corrales et al., 2014a; Catala et al., 2011). Drought stress tolerance tests were performed on plants grown in soil in individual pots. After 2 weeks, water supply was cut off for 15 days and then watering was resumed during 10 days. Plant survival rates and fresh weights were measured 10 days after the re-watering period. Freezing tolerance was analyzed by exposing non-acclimated or cold-acclimated ( 7 days at $\left.4^{\circ} \mathrm{C}\right) 2$-week-old plants to -5 and $-6^{\circ} \mathrm{C}$ or -9 and $-10^{\circ} \mathrm{C}$ for $6 \mathrm{~h}$, respectively. Tolerance was determined as the capacity of plants to resume growth 2 weeks after returning to control conditions.

\section{Microarray analysis}

Genome-wide expression studies with ATH1 array (Affymetrix) were performed using 3-week-old 35S::AtCDF3 and Col-0 plants, grown in chambers under $21 / 18 \stackrel{\circ}{ } \mathrm{C}$, 16/8 $\mathrm{h}$ photoperiod. Three biological replicates (leaves) were harvested at zeitgeber time zero (ZTO) when lights were switched on and frozen into liquid $\mathrm{N}_{2}$ for RNA extraction. Arrays for the different plant materials were hybridized according the Affymetrix GeneChip Expression Analysis manual (www.affymetrix.com). Differentially expressed genes in 35S::CDF3 compared to WT Arabidopsis plants were selected (1.5-fold; $P$ value $<0,05)$. They were functionally annotated by search in the TAIR Arabidopsis database, analysed using the e-northern expression browser tool (http://bar.utoronto.ca/affydb/cgi-bin/affy $\mathrm{db}$ exprss browser in.cgi; Toufighi et al., 2005) and listed in Supplemental table S1. Venn diagrams were performed using Venny software (http://bioinfogp.cnb.csic.es/tools/venny; Oliveros, 2007). Gene Ontology analyses were performed using agriGO 
(http://bioinfo.cau.edu.cn/agriGO/; Du et al., 2010) and REVIGO (http://revigo.irb.hr/; Supek et al., 2011) software.

Pairwise comparisons were made using datasets of differentially regulated genes in gi-100, cdf1235 mutants (Fornara et al., 2015) and 35S::CDF3 plants and publicly available data of cold- and drought-regulated genes in Arabidopsis (Matsui et al., 2008). Venn diagrams were performed using Venny tools (http://bioinfogp.cnb.csic.es/tools/venny; Oliveros, 2007). For more details see Appendix S1.

\section{Metabolomic analyses}

Non-targeted and targeted metabolomics analyses were performed on 12-day-old control plants (Col-0) and two independent 35S::CDF3 lines. Samples were harvested at ZTO. Extraction, manipulation and mass spectrometric analysis of samples followed an adapted protocol described in Corrales et al., (2014a). For more details, see Appendix S1.

\section{RESULTS}

\section{Expression patterns of CDF3 suggest its participation in abiotic stress} responses in vegetative tissues of Arabidopsis

Previously, we have identified a group of tomato DOF TFs (SICDFs) that exhibit specific expression patterns in response to diverse environmental stresses and display functions related to abiotic stress tolerance and flowering time (Corrales et al., 2014a). In order to identify DOF genes that could be involved in the regulation of 
plant responses to different abiotic stresses in Arabidopsis, we examined expression patterns of the complete set of Arabidopsis DOF-encoding genes in plants exposed to drought, salinity, osmotic, extreme temperatures or oxidative stresses using transcriptomic data available from public databases. We found that DOFs genes of D group are differentially expressed in different vegetative tissues such as roots and leaves in response to some of the treatments (Fig. S1). Interestingly, among them the set of Cycling Dof Factors (CDF1-5) was particularly highly induced under some of those stresses. The strong and fast response of CDF3 indicated that this TF might be a regulator of abiotic stress responses in Arabidopsis. In this study the function of CDF3 was further characterized.

To confirm that $C D F 3$ expression is controlled by different environmental cues, we performed detailed qRT-PCR expression analyses using RNA isolated from 3-weekold Arabidopsis plants that had been subjected to different abiotic stresses such as salinity, high and low temperatures, dehydration and also to exogenous $A B A$ treatments for different periods of time. Transcript levels of $C D F 3$ in leaf tissues are significantly increased in response to temperature stress, dehydration, salinity, and exogenous ABA treatment although with different dynamics and extents (Fig.1ab). Higher levels of CDF3 transcripts were observed in response to extreme temperatures, dehydration and $\mathrm{ABA}$ treatment, showing an earlier induction in response to dehydration and reaching maximum levels at $4 \mathrm{~h}$. By contrast, induction of $C D F 3$ was also observed in leaf tissues under salt treatment, with a retarded profile, reaching maximum levels at $24 \mathrm{~h}$.

In order to perform a deeper analysis of the spatial expression patterns of CDF3 in response to abiotic stress, a $1-\mathrm{kb}$ region upstream of the CDF3 transcription start site was fused to the uidA coding sequence to generate the $p C D F 3:: G U S$ reporter 
that was transformed into wild-type plants (WT). A significant GUS staining was detected in vascular systems of leaves and stems, guard cells, pollen and petals (Fig. 1c). Interestingly, the CDF3 promoter also produced a strong signal in mature seeds, showing maximum levels of expression at a later maturation stage as compared to the GUS staining pattern observed for the seed CRUCIFERIN gene (pCRU::GUS, Fig. 1e; Suzuki et al., 2001). Further qRT-PCR experiments confirmed the observed CDF3 expression profile (Fig. 1d). When 3-weeks-old pCDF3::GUS transgenic plants were exposed to different abiotic stresses such as low and high temperatures, dehydration and $\mathrm{ABA}$ or high salt treatments, GUS expression increased with very similar patterns in all cases, regardless of the treatment. GUS staining was detected in leaves, stems as well as main and lateral roots, being especially strong in vascular bundles (Fig. 1f). All these data indicate that the expression of CDF3 is dynamic during plant development, and also in response to different abiotic stresses and that its regulation occurs at least partly at the transcriptional level.

\section{CDF3 protein localizes to the cell nucleus and displays specific DNA-binding and activation properties}

To investigate the subcellular localization of the CDF3 protein, different translational fusions of the ORF to the C-terminus of GFP were made. These constructs, driven by the 35S CaMV promoter, were used for transient expression assays by particle bombardment of onion epidermal cells and for transformation of Arabidopsis plants. Figure $2 \mathrm{a}$ shows that the GFP-CDF3 fusion protein was mainly localized in the nuclei of onion epidermal cells. In contrast, the GFP control was observed in both nuclei 
and cytoplasm of these cells. Similar results were obtained in stable transgenic plants (Fig. 2b).

To gain deeper understanding of CDF3 function, we analyzed its activation properties in transient expression assays in Arabidopsis protoplasts. To this end, effector plasmids containing Arabidopsis CDF3 driven by the $35 S$ promoter (35S::CDF3), and the previously characterized tomato SICDF3 (35S::SICDF3; Corrales et al., 2014a) as positive control, were co-transfected with a reporter plasmid that contains the reporter LUCIFERASE gene (LUC) under control of a minimal promoter containing either the native DOF cis acting element (p4XDOF::LUC) or its mutagenized version (p4xDOFmut::LUC). The results shown in Figure 2C, indicated that AtCDF3 and SICDF3 promoted LUC reporter gene expression of the construct harboring the native DOF cis acting element $5^{\prime}$-AAAG-3', whereas they could not activates the expression of LUC when using the construct

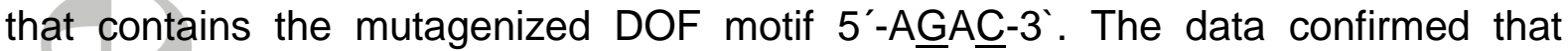
CDF3 could bind specifically to the 5'-AAAG-3' cis-DNA element, and also that moderately activates the LUC reporter gene as the tomato homologous gene SICDF3 (Fig. 2c; Corrales et al., 2014a).

\section{Overexpression of CDF3 enhances drought and low temperature tolerance in}

\section{Arabidopsis}

The presented in silico expression analyses suggested that CDF3 might play an important role in plant responses to different abiotic stresses. To further explore this possibility, a phenotypic characterization of CDF3 gain and loss of function plants was performed by analyzing their responses under drought and osmotic stress 
conditions. Arabidopsis plants overexpressing the full length CDF3 under control of the 355 promoter were generated, and two homozygous lines with relatively high expression levels of CDF3 were selected for further analyses (Fig. 3a). In addition, we identified a T-DNA insertion mutant cdf3-1 (GK808G05) with the insertion site located at position 792 from the ATG, in the middle of the DOF DNA binding domain according to the genome sequence and disruption verified by the absence of CDF3 expression (Fig. S2). When grown in soil under standard greenhouse conditions, cdf3-1 plants did not show apparent developmental differences relative to WT control plants (Col-0). Nevertheless, CDF3 overexpressing plants flowered slightly later than WT control plants under LD conditions (Fig. S3). Interestingly, similar results were previously reported by Fornara et al., (2009) when CDF3 is overexpressed in companion cells using the SUCROSE TRANSPORTER 2 (SUC2) promoter. In addition, when plants were subjected to water deprivation for 15 days and allowed to recover for 10 days during which they were watered, WT and cdf3-1 plants exhibited similar severe symptoms of water loss and significant wilting. In contrast, 35S::CDF3 transgenic plants were less affected, keeping healthy greener leaves. In fact, after a 10 days recovery period, $35 S:: C D F 3$ transgenic plants exhibited better survival rates and higher fresh weight than WT and cdf3-1 plants (Fig. 3bc). These plants were also evaluated for osmotic stress tolerance in different germination and root elongation assays. We followed germination and appearance of green cotyledons in 35S::CDF3, cdf3-1 and WT seeds germinated on 1/2MS (control) or 1/2MS supplemented with 200 or $250 \mathrm{mM}$ mannitol by giving them scores after 3 and 5 days, respectively. When sown on control MS medium, all genotypes displayed similar germination behavior, but in the presence of 200 or $250 \mathrm{mM}$ mannitol, the germination rates were clearly higher and cotyledons greener in $355:: C D F 3$ 
compared to WT plants, and they scored significantly lower in cdf3-1 plants (Fig. 3d). In a second experiment, primary root elongation assays were conducted for 35S::CDF3, cdf3-1, and WT plants grown either on 1/2MS medium (control) or 1/2MS medium supplemented with $200 \mathrm{mM}$ mannitol for 10 days (Fig. 3e). To evaluate growth differences between plants under control and osmotic stress conditions, data were represented as the percentage of root growth relative to standard conditions. Under control conditions, there was no difference between gainand loss- of function lines and WT plants. In contrast, when grown on osmotic stress medium, 35S::CDF3 lines showed moderate but statistically significant higher values of relative root growth than WT plants, whereas cdf3-1 plants exhibited lower values of relative root growth (Fig. 3e-f).

Since low temperatures rapidly induce the expression of $C D F 3$, we decided to investigate whether this protein could have a role in tolerance to freezing temperatures. With this purpose, the freezing tolerance of CDF3 gain- and loss-offunction plants was analyzed before and after cold acclimation for $7 \mathrm{~d}$ at $4^{\circ} \mathrm{C}$. Freezing tolerance was determined in non-acclimated and cold-acclimated plants as their capacity to resume growth after being exposed for $6 \mathrm{~h}$ to different freezing temperatures when returned to control conditions. Interestingly, when compared to non-acclimated WT plants CDF3 overexpressing plants show higher levels of freezing tolerance, whereas, cdf3-1 mutants display significant lower tolerance to freezing (Fig. 4a). Moreover, after cold acclimation 35S::CDF3 lines are also notably more freezing tolerant than WT plants (Fig. 4b), while cdf3-1 plants are impaired in their capacity to tolerate freezing. The freezing tolerance phenotypes of nonacclimated and cold-acclimated WT, cdf3-1 and 35S::CDF3 plants are displayed in Fig. $4 \mathrm{~cd}$, respectively, as a representative example. These data indicate that CDF3 
acts as a positive regulator of constitutive freezing tolerance and cold acclimation response in Arabidopsis.

\section{Enhanced photosynthetic capacity of CDF3 overexpressing plants under} osmotic stress conditions.

To investigate the underlying mechanisms involved in the response of 35S::CDF3 and cdf3-1 plants to dehydration we examined different physiological parameters such as net photosynthesis and related gas exchange variables, stomatal conductance and sub stomatal $\mathrm{CO}_{2}$ concentration using an LI-6400 infrared gas analyzer (LICOR). Three-week-old 35S::CDF3 (L2.1), cdf3-1 and WT plants were transplanted to hydroponic culture to facilitate osmotic stress treatment with $5 \%$ PEG-8000 for 24 hours, after which photosynthesis parameters were measured and represented as percentage to untreated control conditions. Interestingly, plants grown under osmotic stress conditions displayed genotype-dependent changes in photosynthetic rates that were not observed under control conditions. As shown in Fig. $3 g$, photosynthetic rates are higher in CDF3 overexpressing plants and lower in cdf3-1, respectively. A similar response was observed for the stomatal conductance $\left(g_{s}\right)$ (Fig. 3g). Furthermore, the higher increase in the substomatal $\mathrm{CO}_{2}$ concentration in the control plants (292 to $315 \mu \mathrm{mol} / \mathrm{mol}$ ) and cdf3-1 (297 to $316 \mu \mathrm{mol} / \mathrm{mol}$ ) compared to $35 S:: C D F 3$ plants (294 to $300 \mu \mathrm{mol} / \mathrm{mol}$ ) under osmotic stress suggests higher biochemical limitations to photosynthesis in the lines with normal or compromised levels of CDF3. Accordingly, we observed a reduction in the maximum quantum yield of PSII $\left(F_{v} / F_{m}\right)$ in cdf3-1 and WT plants, which indicates the existence of photo-inhibition events, whereas this parameter was not affected by osmotic treatment in 35S::CDF3 plants (Fig. S4). The higher photosynthetic performance of 
the CDF3 overexpressing plants under osmotic stress conditions supports the higher growth observed in these plants under water stress.

Since stomatal conductance is greatly affected by $A B A$, we decided to investigate the possible role of $A B A$ in the different responses of the stomatal conductance observed in the analyzed lines. Thus, 4-week-old 35S::CDF3 (L2.1), cdf3-1, and control (Col-0) plants grown in soil were analyzed by spraying with $0.5 \mu \mathrm{M} A B A$ solution in the underside of the leaves and photosynthesis parameters were measured 1, 2 and $3.5 \mathrm{~h}$ after treatment. The results obtained revealed that these lines showed significant differences in photosynthetic parameters with different dynamics and extents (Fig. 3h). While control and cdf3-1 plants exhibited a similar significant reduction of stomatal conductance values after 1 hour of $A B A$ treatment (60\% of the non-treated), 35S::CDF3 overexpressing plants exhibited a delayed response with almost no effect after 1 hour of the treatment. However at longer times (2-3h), 35S::CDF3 plants finally reach stomatal conductance values similar to WT and cdf3-1 plants. Accordingly, the photosynthetic rate followed a similar response, showing an earlier decrease (0-2 hours) in control and loss-of-function plants, and delayed in 35S::CDF3 plants, although equaled to the former ones after 3.5 hours. Taken together, our data suggest that lower biochemical and stomatal limitations to photosynthesis results from high levels of $C D F 3$ in overexpressing plants and this may lead to bigger size under abiotic stress conditions.

\section{Transcriptome analysis of transgenic Arabidopsis overexpressing CDF3}

To further gain insight into the molecular mechanisms underlying the higher tolerance to drought and low temperatures associated to the CDF3 overexpression, transcriptome analyses of three-week-old 35S::CDF3 (L2,1) and Col-0 plants were 
performed using the Affymetrix Arabidopsis oligo microarray and analyzed at ZTO. The results of the study reveal that among $\sim 24,000$ Arabidopsis genes 531 were differentially expressed (>1.5-fold change; $\mathrm{P}$ value $\leq 0,05)$ in $C D F 3$ overexpressing plants compared with WT plants in control conditions (Fig. 5; Table S1). About, twothirds of the genes (409) were up-regulated, whereas 122 were down-regulated. Moreover, Gene Ontology annotation analyses of the misregulated genes in $35 S:: C D F 3$ plants revealed that the putative targets of CDF3 are highly enriched in stress-related and signal transduction categories, like "response to water deprivation", "light intensity", "cold", "oxidative stress" and metabolism, like "amino acid" and "carbohydrate biosynthesis" (Fig. 5c,d), thus indicating a role of CDF3 in early stress responses. Among the upregulated genes a group of LEA (Late Embryogenesis Abundant protein), HSP (Heat Shock Protein) and DNAJ genes (Table S1) that function in osmotic stress regulation, protein folding and assembly processes, autophagy and protection of cellular structural integrity under extreme temperatures, osmotic and dehydration conditions (Ingram and Bartels 1996; Chen et al., 2010; Sato \& Yokoya, 2008; Yang et al., 2015) were misregulated in 35S::CDF3 plants, indicating the participation of CDF3 in osmoprotection. Using the e-northern Expression Browser tool (Toufighi et al., 2005), we performed a detailed classification of the identified genes that revealed that many of them are regulated by different abiotic stresses (Fig. 5a). In fact, among the up-regulated genes 337, 109, 147 and 76 were significantly misregulated $(>1.5$-fold) in at least one time-point during drought, low temperature, salinity and osmotic stresses, respectively (Fig. 5a). Remarkably among them is included a group of genes previously reported to be involved in cold and drought stress responses, such as cold-regulated-genes COR78/RD29A, COR15a, COR413, KIN1 and EARLY RESPOSIVE TO 
DEHYDRATION (ERD-7,-10,-12 and -15), GALACTINOL SYNTHASE (GOLS2) and the SUCROSE SYNTHASE 1 (SUS1) gene (Déjardin et al., 1999; Kim and Nam, 2010; Kiyosue et al., 1994; Thomashow, 2010; Taji et al., 2002; Table S1). Thus, these data suggest that CDF3 might function in the regulation of cellular integrity, metabolism and oxidative ROS homeostasis to control cellular and oxidative damage promoted by drought and low temperatures.

A detailed exploration of the previously described CDF3 regulon of 531 target genes allowed the identification of important genes encoding key regulatory transcription factors reported to participate in different abiotic stress responses: DREB2A (Sakuma et al., 2006), WRKY46 (Ding et al., 2014), ERF6 (Dubois et al., 2013) and WRKY30 (Scarpezi et al., 2013) involved in drought and osmotic stress signaling, CBF1, CBF2, CBF3 (Liu et al., 1998; Medina et al.,1999; Novillo et al., 2007) and ZAT6 (Shi et al., 2014) involved in low temperature stress, and ZAT10 and ZAT12 (Davletova et al., 2005; Mittler et al., 2006) involved in oxidative stress. These results indicate that CDF3 might play an important role organizing abiotic stress responses by controlling the expression of key stress-related transcription factors. Quantitative RT-PCR was performed to confirm some of the identified differentially expressed genes in the 35S::CDF3 plants. In this analysis we included both classical abiotic stress-responsive genes such as COR15A, RD29A and ERD10 and the transcriptional regulators $C B F 1, C B F 2, C B F 3, Z A T 10, Z A T 12$ and DREB2A. Figure $6 a b$ shows the expression levels of the analyzed genes in $35 S:: C D F 3$ transgenic lines, where they exhibit higher values (from two- to four-fold) than in the WT plants. These data confirmed the results of the chip experiments and indicate that CDF3 might be an upstream activator in drought and low temperature stress pathways, 
acting directly or indirectly on the expression of different stress-regulated target genes.

It has been reported that mutations in $G /$ increased CDF abundance preventing the expression of $\mathrm{CO}$ and $\mathrm{FT}$, promoting late flowering and the increase tolerance to oxidative stress (Fornara et al., 2009, 2015). By contrast, the multiple mutant gi-100cdf1235 suppresses late flowering, oxidative stress tolerance of gi and restores expression patterns of $C O$ and FT. To study the overlap of the GI-CDF module and determine the specific contribution of CDF3, we compared the datasets of the differentially expressed genes in gi and cdf1235 mutants obtained from non-stressed plants (Fornara et al., 2015) with the ones from 35S::CDF3 plants. In the case of the gi mutant, whose CDFs appear upregulated, we found that among the misregulated genes about $12 \%$ were also differentially expressed in $35 S:: C D F 3$ plants (Table S12, Fig. S5). Notably, we observed that a large set of the genes that are common between 35S::CDF3 and gi transcriptional profiles, about 83.4\% (Table S3), are upregulated, which could be expected considering that both lines present high levels of expression of CDF3. A similar comparison with the cdf1235 multiple mutant, shows that a limited number of genes is upregulated in the CDF3 overexpessor and repressed in cdf1235 plants, suggesting that CDF3 might regulate this specific set of genes (Table S3).

To analyze the contribution of the GI-CDF module to drought and low temperature responses and precisely determine the genes that depend on Gl and CDF3, we compared datasets of differentially regulated genes in the gi mutant and $35 S:: C D F 3$ plants, with publicly available data obtained from Arabidopsis plants exposed to drought and low temperature stress treatments (Matsui et al 2008). Notably, a limited overlap between stress-responsive- genes regulated by GI and CDF3 was observed 
(Fig S6). Actually, in the case of 35S::CDF3 plants, $92.6 \%$ and $89.7 \%$ of the drought and low temperature regulated genes identified, respectively, are specific for CDF3, and were not differentially expressed in gi mutant (Fig S6). Similarly, in the gi mutant, about $83 \%$ and $84 \%$ of the drought and low temperature regulated genes identified are not misregulated in CDF3 overexpressor plants (Fig. S6). Altogether, these data suggest that a significant set of genes regulated by the GI-CDF module are under direct control of CDF3, but also that CDF3 specifically modulates the expression of certain genes in a Gl-independent fashion.

To elucidate whether CDF3 might directly regulate abiotic stress responsive genes, we first searched for common cis-acting elements present in the promoters of the CDF3 misregulated genes using the Promomer tool (Toufighi et al., 2005) and found overrepresentation of the DOF DNA-binding motif 5'-T/AAAAG-3' in their promoter regions (Fig. S7). Among these genes the COR15 promoter was selected for further studies as a potential target of CDF3. Using protoplast transformation, a 35S::CDF3 effector plasmid was cotransfected with a reporter plasmid harboring the uidA reporter gene under control of $1 \mathrm{~kb}$ promoter region of COR15 containing multiple DOF cis-DNA binding elements (Fig. S8). As shown in Fig. 6c, CDF3 activates the expression of the reporter gene, most likely through one of the DOF binding sites present in the COR15 promoter. To confirm the potential role of the DOF binding site as an abiotic stress response cis-acting element component, the uidA gene under control of a minimal promoter containing a $2 \times$ DOF cis-DNA element $(p B T 102 \times D O F-$ GUS) was used to transform Arabidopsis protoplasts incubated under different stress conditions such as extreme temperatures $\left(4^{\circ} \mathrm{C}\right.$ and $\left.37^{\circ} \mathrm{C}\right)$ or treated with $\mathrm{NaCl}$

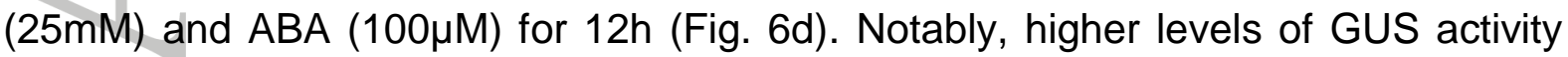
compared to the untreated control were observed under low and high temperature 
treatments (>1,5-fold), slightly higher levels were detected under salt treatment and no differences were found in ABA treatments. These results obtained indicate that

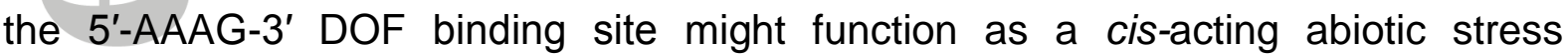
response element and supports the role of the CDFs as candidate nuclear transacting factors operating on it.

\section{The overexpression of CDF3 in vegetative tissues impacts sugar and amino acid metabolism}

Drought and extreme temperatures are conditions that promote substantial changes in plant physiology and metabolism (Rizhsky et al., 2004; Seki et al., 2007; Chaves et al., 2009). To investigate whether CDF3 overexpression in Arabidopsis promotes changes in the plant metabolome that would be consistent with the higher drought tolerance, we performed metabolomic analyses of these plants. In a first step, a nontargeted metabolite analysis of 35S::CDF3 (lines L2.1 and L5.4) and WT plants was carried out. The different samples were compared by principal component analysis (PCA) considering about 1000 molecular features per sample. The results revealed that both 35S::CDF3 overexpressor lines exhibited a significant alteration of the metabolome (Fig. 7a). To further dissect these changes we performed a targeted metabolomic profiling by gas chromatography-mass spectrometry (GC-MS) to study the relative levels of different polar compounds, including proteinogenic amino acids as well as other amino acids and distinct sugars, extracted from 12-day-old WT and 35S::CDF3 plants grown under non-stress conditions. As shown in Fig. 7c and Table S4, comparison of the GC-MS profiles revealed a number of clear differences between WT and overexpressing lines. Overexpression of CDF3 in Arabidopsis 
significantly induced the accumulation of sugars like sucrose (1.1-fold) and glucose (2-fold) and amino acids like L-leucine (1.3-fold), L-asparagine (1.82-fold), Lglutamine (1.53-fold), y-aminobutyric acid (GABA; 1.3-fold) and L-proline (2.2-fold), previously associated to stress tolerance and indicative of increased nitrogen assimilation, as reported for other DOF genes such as ZmDOF1 (Yanagisawa et al., 2004).

\section{DISCUSSION}

During the last decade different reports have implicated the DOF transcription factors in the regulation of biological processes related to plant growth and development. In this work we have identified a group of Arabidopsis DOF genes known as CDFs whose expression responds to different abiotic stresses like salt, drought, and extreme temperatures. The results of our study provide functional evidence in support that one of them, CDF3, contributes to processes such drought, osmotic and cold stress tolerance and flowering. CDF3 displays spatially separated functions modulating the expression of different sets of genes that operate in both GIGANTEAdependent and -independent pathways.

\section{Novel disclosed functions of CDF3 in abiotic stress responses}

In this work we analyzed the expression patterns of the complete set of 36 genes encoding DOF proteins of Arabidopsis (Lijavetzky et al., 2003), and found that those included in the group D, are highly expressed in response to different abiotic stress conditions. Interestingly, among them, the CDFs seemed to be regulated by drought, salinity and extreme temperatures. However, under stress conditions they display dissimilar patterns in timing of response and in spatial expression in roots and 
shoots, suggesting a distinct participation in specific responses to environmental changes.

To further explore this aspect, we have performed a detailed expression analysis of CDF3 using promoter-GUS fusions and qRT-PCR, and observe that it is rapidly induced in leaves and roots in response to different abiotic stresses, with similar expression patterns regardless of the treatment. This behavior indicates that CDF3 may respond to an intermediate common effector shared by the different stress treatments. Moreover, the observation that under abiotic stress conditions CDF3 extends its expression pattern to leaves and roots from the vascular tissues, implies that it might play additional roles under non-stress conditions, as reported for its involvement in the control of flowering. In addition, it might suggest that CDF3 functions are spatially separated, by which flowering time is controlled in the companion cells of the phloem, whereas stress responses might take place in alternative tissues. Consistently, the flowering regulators, $\mathrm{CO}$ and FT, two direct targets of CDFs are controlled precisely in the vasculature of leaves (Fornara et al., 2009), suggesting that CDFs might display additional functions related to abiotic stress responses through alternative targets or the interaction with different factors in other tissues.

To clarify the specific participation of CDF3 in response to abiotic stress conditions we performed a functional characterization of Arabidopsis gain- and loss-of-function mutants. Phenotypic analyses, including survival rates and root length assays under stress conditions, showed that the CDF3 T-DNA insertion mutant displays reduced tolerance to drought and low temperatures. In contrast, CDF3 overexpressing lines are more tolerant to drought, osmotic and low temperatures, indicating that CDF3 plays multiple roles to confer protection from different abiotic stresses. This finding is 
also supported by recent work reporting that a multiple cdf1235 mutant exhibits higher sensitivity to oxidative stress promoted by methylviologen (Fornara et al., 2015). However, the authors observed increased susceptibility to low temperature stress in the cdf1235 mutant mainly in the gi background, suggesting a complex interaction between $\mathrm{Gl}$ and the CDFs, and that GI might need the participation of the CDFs, or a specific CDF member, to play some of its roles in response to low temperatures. The work presented here extends Fornara's observations and provides functional evidence that CDF3 plays a key role in plant responses to abiotic stresses such as low temperature, osmotic and drought, both through GI-dependent and -independent pathways.

The transcriptional profiling performed in this study revealed that about half of the differentially expressed genes in $35 S:: C D F 3$ are related to responses to osmotic, drought or extreme temperatures, which is in agreement with the phenotypes displayed by the CDF3 overexpressing plants under abiotic stress conditions. Interestingly, CDF3 overexpression upregulates a group of genes encoding LEAs, HSPs and DNAJ proteins that have been involved in osmotic regulation, protein folding, autophagy and protection of cellular structures under abiotic stresses (Ingram and Bartels, 1996; Wang et al., 2004; Chen et al., 2010), in support of a role of CDF3 in the regulation of protective mechanisms against cellular damage caused by osmotic and low temperature stress. These results are in agreement with previous data of the gi mutant showing increased stability and accumulation of CDF proteins, expression of COR stress-regulated genes and increased protection to low temperatures (Fornara et al., 2015). However, the transcriptomic analyses reported here showed a limited overlap between stress-responsive-genes regulated by GI and CDF3. It is worth mentioning that sampling of plant materials of the $35 S:: C D F 3$ and 
gi mutants for transcriptomic analyses (this work and Fornara et al., 2015) was done at ZT0 and ZT12 respectively, when native CDF3 and GI mRNA expression levels in the corresponding controls are most contrasting. Although this fact may reduce a partial overlap of commonly regulated genes, the presented results support that GI and CDF3 are involved in multiple abiotic stress responses, and display specific functions in drought and low temperatures stresses, most likely by controlling the expression of different sets of genes involved in organ-specific stress responses.

Metabolic profiling of CDF3 overexpressing plants revealed an increase of amino acids like proline and GABA, and sugars like sucrose and glucose, usually accumulated at higher levels in plant tissues exposed to extreme temperatures, osmotic stress or drought (Rizhsky et al., 2004; Gill and Tuteja, 2010; Hussain et al., 2011). These metabolites function in osmotic adjustment, protection of membranes and ROS scavenging (Rajasekaran et al., 2000; Claussen, 2005; Munns and Tester, 2008; Farrant and Moore, 2011) and their increased levels are in agreement with the higher tolerance to abiotic stress displayed by the CDF3 overexpressing plants. This finding is also supported by recent work of Fornara et al. (2015) reporting that a multiple cdf1235 mutant exhibits higher sensitivity to oxidative stress and reduced expression of several cold-regulated genes. Overall, our results demonstrate the participation of CDF3 in plant responses to different abiotic stress conditions, and also that individual CDFs might regulate specific target genes in response to particular environmental perturbations. 


\section{Impact of CDF3 on carbon and nitrogen metabolism}

The expression analysis of $C D F 3$ revealed a complex pattern, being detected in different tissues during development. Particularly, it is highly expressed in organs and tissues with different sink/source activities such as shoots and roots, and reproductive structures like flowers and seeds. This may indicate that CDF3 plays tissue-specific functions by controlling the expression of genes involved in particular metabolic processes. In this respect, metabolite analyses of 35S::CDF3 plants revealed that under control conditions the transgenic lines exhibit important changes, including higher levels of sugars such as sucrose and glucose, and the accumulation

of different amino acids such as glutamine, asparagine, proline and GABA. These observations are in agreement with previously reported metabolomic analyses of sex3 mutant (gi allele) which showed higher levels of several aminoacids, sugars and sugar alcohols relative to wild type (Messerly et al., 2007). Interestingly, the levels of GABA, asparagine and glutamine are reliable indicators of nitrogen use efficiency (Stitt and Krapp, 1999; Yanagisawa et al., 2004; Foyer et al., 2006). In fact, GABA has been involved in nitrogen storage through the pathway that converts glutamate to succinate (GABA shunt), with a great impact in nitrogen economy of plants (Shelp et al., 1999). The observed higher amino acid content in the overexpression lines might be related to an improvement of nitrogen assimilation as previously described for other DOF TFs (Yanagisawa, 2004).

On the other hand, GABA and the GABA shunt in plants have been connected with other functions related to abiotic stress, including osmoregulation (Shelp et al., 1999), cytosolic pH regulation (Snedden et al., 1995), protection against oxidative stress (Bouche et al., 2003) and maintenance of the C/N balance (Shelp et al., 2012; Studart-Guimaraes et al., 2007). Moreover it has been shown that GABA may also 
act as a putative long-distance signal molecule in the regulation of nitrate uptake (Beuve et al., 2004). The metabolite profile data presented here suggests that CDF3 could participate in the regulation of the $\mathrm{C} / \mathrm{N}$ metabolism favoring plant growth and development under specific stress conditions.

\section{CDF3, a connection between flowering time and abiotic stress responses}

The data presented in this work confirm the previously reported participation of CDFs in the control of flowering time. Precisely, the overexpression of $A t C D F s$ in phloem companion cells (Imaizumi et al., 2005; Fornara et al., 2009) or the constitutive overexpression of tomato SICDFs in Arabidopsis (Corrales et al., 2014a) promote a delay in flowering time under LD conditions. Likewise, here we show that the constitutive CDF3 overexpression not only has an impact in flowering time but also in plant responses to different abiotic stresses.

The timing of flowering, alongside with the adaptability to changing environmental conditions, has significant consequences for the reproductive success in plants. Accordingly, plants must closely integrate changes in the environment to determine the onset of flowering and ensure reproductive success. Triggering the transition from vegetative to reproductive phase relies on an extremely intricate network, linking multiple signaling pathways and regulatory proteins (Blümel et al., 2015). Among them, the Gl protein plays a central role in diverse signaling pathways, including circadian clock regulation photoperiodic, sugar and light signaling and stress responses (Fowler et al., 1999; Gould et al., 2006; Mizoguchi et al., 2005;

Park et al., 1999; Cao et al., 2005). Gl activates the expression of the central flowering regulators $C O$ and $F T$, by promoting the degradation of the CDFs 
(Imaizumi et al., 2005; Sawa et al., 2007; Fornara et al., 2009). Interestingly, the gi mutant presents higher stability and accumulation of CDF proteins, and shows increased expression of cold regulated genes and higher tolerance to cold and oxidative stress (Han et al., 2013, Kurepa et al., 1998; Cao et al., 2005; Fornara et al., 2015).

In this context, our results disclose that CDF3 controls the expression of a group of genes involved in plant responses to extreme temperatures, drought and osmotic stress, including several central abiotic stress regulators like CBFs, DREB2A and ZAT12. Interestingly, the overexpression of these TFs not only promotes changes in the response to different abiotic stresses but also results in late flowering (Gilmour et al., 2004; Vogel et al., 2005; Sakuma et al., 2006; Achard et al., 2005). It has been established previously that CDF3 participates in the control of flowering time through the transcriptional regulation of key factors like CO (Imaizumi et al., 2005; Sawa et al., 2007; Fornara et al., 2009). However, our results highlight its relation to drought and cold response pathways, and the regulatory action on CBF/DREB2A-CRT/DRE and ZAT12 modules with an impact on flowering time as well.

Finally, our metabolomic analyses reveal that CDF3 overexpresssion promotes important changes in the plant metabolome, altering the levels of specific compounds with protective functions that alleviate detrimental effects of abiotic stress conditions. These results also would allow us to hypothesize that CDF3 might regulate the partition of $\mathrm{C} / \mathrm{N}$ rich compounds depending on age, stage of plant development and environmental cues, and eventually influence the control of flowering time. 
In conclusion, the present study provides new notions about the function of DOF TFs and unveils $\mathrm{CDF} 3$ as a key factor that display multiple roles related to plant responses to adverse environmental conditions and the developmental program underlying the transition from vegetative to reproductive phase.

\section{ACKNOWLEDGEMENTS}

We thank Dr. Pablo González-Melendi and Dr. Jan Zouhar for technical handling of the confocal microscope and Dr. Rafael Catalá for the assistance with the low temperature stress assays. This work was supported by grants from Instituto Nacional de Investigación y Tecnología Agraria y Alimentaria (INIA; projects 20090004-C01, 2012-0008-C01), Spanish Ministry of Science and Innovation (projects BIO2010-1487, BFU2013-49665-EXP). ARC and JDF were supported by INIA predoctoral fellowships. 


\section{REFERENCES}

Achard P., Gong F., Cheminant S., Alioua M., Hedden P. \& Genschik P. (2005) The cold-inducible CBF1 factor-dependent signaling pathway modulates the accumulation of the growth-repressing DELLA proteins via its effect on gibberellin metabolism. Plant Cell 20, 2117-2129.

Ahuja I., de Vos R.C., Bones A.M. \& Hall R.D. (2010) Plant molecular stress responses face climate change. Trends Plant Science 15, 664-674.

Alonso R., Oñate-Sánchez L., Weltmeier F., Ehlert A., Diaz I., Dietrich K., VicenteCarbajosa, J. \& Dröge-Laser W. (2009) A Pivotal Role of the Basic Leucine Zipper Transcription Factor bZIP53 in the Regulation of Arabidopsis Seed Maturation Gene Expression Based on Heterodimerization and Protein Complex Formation. Plant Cell 21, 1747-1761.

Beuve N., Rispail N., Laine P., Cliquet J.B., Ourry A. \& Le Deunff E. (2004) Putative role of $\mathrm{y}$-aminobutyric acid (GABA) as a long-distance signal in up-regulation of nitrate uptake in Brassica napus L. Plant Cell Environment 27, 1035-1046.

Blümel M., Dally N. \& Jung C. (2015) Flowering time regulation in crops - what did we learn from Arabidopsis? Current Opinion in Biotechnology 32: 121-129

Bouché N., Fait A., Bouchez,D., Møller S.G. \& Fromm H. (2003) Mitochondrial succinic-semialdehyde dehydrogenase of the gamma-aminobutyrate shunt is required to restrict levels of reactive oxygen intermediates in plants. Proc. Natl. Acad. Sci. USA 100, 6843-6848.

Cao S., Ye,M. \& Jiang S. (2005) Involvement of GIGANTEA gene in the regulation of 
the cold stress response in Arabidopsis. Plant Cell Reports 24, 683-690.

Catala R., Medina J. \& Salinas J. (2011) Integration of low temperature and light signaling during cold acclimation response in Arabidopsis. Proc. Natl. Acad. Sci. USA. 19, 16475-16480.

Chaves M.M., Flexas J. \& Pinheiro C. (2009) Photosynthesis under drought and salt stress: regulation mechanisms from whole plant to cell. Annals of Botany 103, $551-560$.

Chen H., Hwang J.E., Lim C.J., Kim D.Y., Lee S.Y. \& Lim C.O. (2010) Arabidopsis DREB2C functions as a transcriptional activator of HsfA3 during the heat stress response. Biochemical and Biophysical Research Communications 401, 238-244.

Chen K.M., Holmström M., Raksajit W., Suorsa M., Piippo M. \& Aro E.M. (2010) Small chloroplast-targeted DnaJ proteins are involved in optimization of photosynthetic reactions in Arabidopsis thaliana. BMC Plant Biololgy 43, 14712229

Claussen W. (2005) Proline as a measure of stress in tomato plants. Plant Science $168,241-248$.

Clough S.J. \& Bent A.F. (1998) Floral dip: a simplified method for Agrobacteriummediated transformation of Arabidopsis thaliana. Plant Journal 16, 735-743.

Corrales A-R., Carrillo L., Nebauer S.G. Renau-Morata B., Sánchez-Perales M., Fernández-Nohales P., Marqués J., Granell A., Pollmann S., Vicente-Carbajosa J., Molina R.V. \& Medina, J. (2014b) Salinity Assay in Arabidopsis. Bio-protocol 4: e1216. 
Corrales A-R., Nebauer S.G., Carrillo L., Fernández-Nohales P., Marqués J., RenauMorata B., Granell A., Pollmann S., Vicente-carbajosa J., Molina R-S. \& Medina, J. (2014a) Characterization of tomato Cycling Dof Factors reveals conserved and new functions in the control of flowering time and abiotic stress responses. Journal of Experimental Botany 65, 995-1012.

Davletova S., Schlauch K., Coutu J. \& Mittler R. (2005) The Zinc-Finger Protein Zat12 plays a central role in reactive oxygen and abiotic stress signaling in Arabidopsis. Plant Physiololgy 139, 847-856.

Déjardin A., Sokolov L.N. \& Kleczkowski L.A. (1999) Sugar/osmoticum levels modulate differential abscisic acid-independent expression of two stressresponsive sucrose synthase genes in Arabidopsis. Biochem Journal 2, 503-509.

Ding Z., Yan J., Xu X., Yu D., Li, G., Zhang S. \& Zheng S. (2014) Transcription factor WRKY46 regulates osmotic stress responses and stomatal movement independently in Arabidopsis. Plant Journal 79, 13-27.

Dubois M., Skirycz A., Claeys H., Maleux K., Dhondt S., De Bodt S., VandenBossche R., De Milde L., Yoshizumi T., Matsui M. \& Inzé D. (2013). Ethylene Response Factor 6 acts as a central regulator of leaf growth under water-limiting conditions in Arabidopsis. Plant Physiology 162, 319-332.

Du Z., Zhou X., Ling Y., Zhang Z. \& Su Z. (2010) agriGO: a GO analysis toolkit for the agricultural community. Nucleic Acids Research 38: 64-70.

Farrant J.M. \& Moore J.P. (2011) Programming desiccation-tolerance: from plants to seeds to resurrection plants. Current Opinion in Plant Biology 14, 340-345. 
Fornara F., Montaigu A., Sánchez-Villarreal A., Takahashi Y., van Themaat E.V.L., Huettel B., Davis S.J. \& Coupland G. (2015) The GI-CDF module of Arabidopsis affects freezing tolerance and growth as well as flowering. Plant Journal 81, 695706.

Fornara F., Panigrahi K.C.S., Gissot L., Sauerbrunn N., Rühl M., Jarillo J.A. \& Coupland G. (2009). Arabidopsis DOF transcription factors act redundantly to reduce CONSTANS expression and are essential for a photoperiodic flowering response. Developmental Cell 17, 75-86.

Fowler S., Lee K., Onouchi H., Samach A., Richardson K., Coupland G. \& Putterill J. (1999) GIGANTEA: a circadian clock-controlled gene that regulates photoperiodic flowering in Arabidopsis and encodes a protein with several possible membranespanning domains. EMBO Journal 18, 4679- 4688.

Foyer C.H., Noctor G. \& Verrier P. (2006) Photosynthetic carbon-nitrogen interactions: modelling inter-pathway control and signalling. Ann. Plant Rev. 14, 325-347.

Galmés J., Medrano H. \& Flexas J. (2007) Photosynthetic limitations in response to water stress and recovery in Mediterranean plants with different growth forms. New Phytology 175, 81-93.

Gill S.S. \& Tuteja N. (2010) Reactive oxygen species and antioxidant machinery in abiotic stress tolerance in crop plants. Plant Physiology and Biochemistry 48, 909-930. 
Gilmour S.J., Fowler S.G. \& Thomashow M.F. (2004) Arabidopsis transcriptional activators $\mathrm{CBF} 1, \mathrm{CBF} 2$, and $\mathrm{CBF} 3$ have matching functional activities. Plant Molecular Biology 54, 767-781.

Gould P.D., Locke J.C., Larue C., Southern M.M., Davis S.J., Hanano S., Moyle R., Milich R., Putterill J., Millar A.J. \& Hall A. (2006) The molecular basis of temperature compensation in the Arabidopsis circadian clock. Plant Cell 18, $1177-1187$.

Han Q., Kang G. \& Guo T. (2013) Proteomic analysis of spring freeze-stress responsive proteins in leaves of bread wheat (Triticum aestivum L.). Plant Physiology Biochemistry 63, 236-244.

Hernando-Amado S., González-Calle V., Carbonero P. \& Barrero-Sicilia C. (2012) The family of DOF transcription factors in Brachypodium distachyon: phylogenetic comparison with rice and barley DOFs and expression profiling. BMC Plant Biology 12, 202.

Hong-Feng Z., Yu-Qin Z., Wei W., Hao-Wei C., Qing-Xin S., Yun-Feng L., Ming-Yu Z., Fang W., Bao-Cai Z., Qing L., Wan-Ke Z., Biao M., Yi-Hua Z., Jin-Song Z. \& Shou-Yi C. (2013) The transcription factor AtDOF4.2 regulates shoot branching and seed coat formation in Arabidopsis. Biochemistry Journal 449, 373-88.

Hussain S.S., Kayani M.A. \& Amjad M. (2011) Transcription factors as tools to engineer enhanced drought stress tolerance in plants. Biotechnology Progress 27, 297-306. 
Imaizumi T., Schultz T.F., Harmon F.G., Ho L.A. \& Kay S.A. (2005) FKF1 F-box protein mediates cyclic degradation of a repressor of CONSTANS in Arabidopsis. Science 309, 293-297.

Ingram J. \& Bartels D. (1996) The molecular basis of dehydration tolerance in plants. Annual Review Plant Physiology Plant Molecular Biology 7, 377-403.

Jarillo J.A., Olmo I., Gómez-Zambrano A., Lázaro A., López-González L., Miguel E., Narro-Diego L., Sáez D. \& Piñeiro M. (2008) Photoperiodic control of flowering time. Spanish Journal Agricultural Research 6, 221-244.

Jefferson R.A., Kavanagh T.A. \& Bevan M.W. (1987) GUS fusions: betaglucuronidase as a sensitive and versatile gene fusion marker in higher plants. EMBO Journal 6, 3901-3907.

Jones M.L. (2013) Mineral nutrient remobilization during corolla senescence in ethylene-sensitive and -insensitive flowers. AoB Plants 5, plt023.

Karimi M., Depicker A. \& Hilson P. (2007) Recombinational Cloning with Plant Gateway Vectors. Plant Physiology 145, 1144-1154.

Kim S.Y. \& Nam K.H. (2010) Physiological roles of ERD10 in abiotic stress and seed germination of Arabidopsis. Plant Cell Reports 29, 203-209.

Kiyosue T., Yamaguchi-Shinozaki K. \& Shinozaki K. (1993) Characterization of two cDNAs (ERD11 and ERD13) for dehydration-inducible genes that encode putative glutathione S-transferases in Arabidopsis thaliana L. Febs Letters 335, 189-192. 
Kiyosue T., Yamaguchi-Shinozaki K. \& Shinozaki K. (1994) Cloning of cDNAs for genes that are early-responsive to dehydration stress (ERDs) in Arabidopsis thaliana L.: Identification of three ERDs as HSP cognate genes. Plant Molecular Biology 25, 791-798.

Kurai T., Wakayama M., Abiko T., Yanagisawa S., Aoki N. \& Ohsugi R. (2011) Introduction of the ZmDof1 gene into rice enhances carbon and nitrogen assimilation under low-nitrogen conditions. Plant Biotechnology Journal 9, 826837.

Kurepa J., Smalle J., Montagu M.V. \& Inzé D. (1998) Effects of sucrose supply on growth and paraquat tolerance of the late-flowering gi-3 mutant. Plant Growth Regulators 26, 91-96.

Lijavetzky D., Carbonero P. \& Vicente-Carbajosa J. (2003) Genome-wide comparative phylogenetic analysis of the rice and Arabidopsis Dof gene families. BMC Evolutionary Biology 3, 17-28.

Liu Q., Kasuga M., Sakuma Y., Abe H., Miura S., Yamaguchi-Shinozaki K. \& Shinozaki K. (1998) Two transcription factors, DREB1 and DREB2, with an EREBP/AP2 DNA binding domain separate two cellular signal transduction pathways in drought- and low-temperature-responsive gene expression, respectively, in Arabidopsis. Plant Cell 10, 1391-1406.

Matsui A., Ishida J., Morosawa T., Mochizuki Y., Kaminuma E., Endo TA., Okamoto M., Nambara E., Nakajima M., Kawashima M., Satou M., Kim JM., Kobayashi N., Toyoda T., Shinozaki K., Seki M. (2008) Arabidopsis Transcriptome Analysis 
under Drought, Cold, High-Salinity and ABA Treatment Conditions using a Tiling Array. Plant Cell Physioloy 49, 1135-1149

Medina J., Bargues M., Terol J., Pérez-Alonso M. \& Salinas J. (1999). The Arabidopsis CBF gene family is composed of three genes encoding AP2 domaincontaining proteins whose expression is regulated by low temperature but not by abscisic acid or dehydration. Plant Physiology 119, 463-470.

Messerli G., Nia VP., Trevisan M., Kolbe A., Schauer N., Geigenberger P., Chen J,. Davison AC,. Fernie A. \& Zeeman S. (2007) Rapid Classification of Phenotypic Mutants of Arabidopsis via Metabolite Fingerprinting. Plant Physiology 143, 14841492.

Mittler R. (2006). Abiotic stress, the field environment and stress combination. Trends in Plant Science 11, 15-19.

Mizoguchi T., Wright L., Fujiwara S., Cremer F., Lee K., Onouchi, H., Mouradov A., Fowler S., Kamada H., Putterill J. \& Coupland G. (2005) Distinct roles of GIGANTEA in promoting flowering and regulating circadian rhythms in Arabidopsis. Plant Cell 17, 2255-2270.

Munns R. \& Tester M. (2008) Mechanisms of salinity tolerance. Annual Review Plant Biology 59, 651-681.

Murashige T. \& Skoog F. (1962) A Revised Medium for Rapid Growth and Bio Assays with Tobacco Tissue Cultures. Physiologia Plantarum 15, 473-497.

Nishiyama R., Le D.T., Watanabe Y., Matsui A., Tanaka M., Seki M., YamaguchiShinozaki K., Shinozaki K. \& Tran L.S. (2012) Transcriptome analyses of a salt- 
tolerant cytokinin-deficient mutant reveal differential regulation of salt stress response by cytokinin deficiency. PLoS One 7, e32124.

Noguero M., Atif R.M., Ochatt S. \& Thompson R.D. (2013) The role of the DNAbinding One Zinc Finger (DOF) transcription factor family in plants. Plant Science $209,32-45$.

Novillo F., Medina J. \& Salinas J. (2007) Arabidopsis CBF1 and CBF3 have a different function than CBF2 in cold acclimation and define different gene classes in the CBF regulon. Proc. Natl. Acad. Sci. USA 104, 21002-21007.

Oliveros J.C. (2007) Venny, An interactive tool for comparing lists with Venn's diagrams. http://bioinfogp.cnb.csic.es/tools/venny/index.html.

Oñate-Sánchez L. \& Vicente-Carbajosa J. (2008) DNA-free RNA isolation protocols for Arabidopsis thaliana, including seeds and siliques. BMC Research Notes 1, 93.

Osakabe Y., Kajita S. \& Osakabe K. (2011) Genetic engineering of woody plants: current and future targets in a stressful environment. Physiologia Plantarum 142, $105-117$.

Park D., Somers D., Kim Y., Choy Y., Lim H., Soh M., Kim H., Kay S. \& Nam H. (1999) Control of circadian rhythms and photoperiodic flowering by the Arabidopsis GIGANTEA gene. Science 285, 1579-1582.

Rajasekaran L.R., Aspinall D. \& Paleg L.G. (2000) Physiological mechanism of tolerance of Lycopersicon spp. exposed to salt stress. Canadian Journal Plant Science 80, 151-159. 
Rizhsky L., Liang H., Shuman J., Shulaev V., Davletova S. \& Mittler R. (2004) When defense pathways collide. The response of Arabidopsis to a combination of drought and heat stress. Plant Physiology 134, 1683-1696.

Rosso M.G., Li Y., Strizhov N., Reiss B., Dekker K. \& Weisshaar, B. (2003) An Arabidopsis thaliana T-DNA mutagenized population (GABI-Kat) for flanking sequence tag-based reverse genetics. Plant Molecular Biology 53, 247-259.

Rueda-López M., Crespillo R., Cánovas F.M. \& Avila C. (2008).Differential regulation of two glutamine synthetase genes by a single Dof transcription factor. Plant Journal 56, 73-85.

Sakuma Y., Maruyama K., Osakabe K., Qin F., Seki M., Shinozaki K. and Yamaguchi-Shinozaki K. (2006) Functional analysis of an Arabidopsis transcription factor, DREB2A, involved in drought-responsive gene expression. Plant Cell 18, 1292-1309.

Sato Y. \& Yokoya S. (2008) Enhanced tolerance to drought stress in transgenic rice plants overexpressing a small heat-shock protein, sHSP17.7. Plant Cell Reporter 27, 329-334.

Sawa M., Nusinow D.A. Kay, S.A. \& Imaizumi T. (2007) FKF1 and GIGANTEA complex formation is required for day-length measurement in Arabidopsis. Science 318, 261-265.

Scarpeci T., Zanor M., Carrillo N., Mueller-Roeber B. \& Valle E. (2008) Generation of superoxide anion in chloroplasts of Arabidopsis thaliana during active 
photosynthesis: a focus on rapidly induced genes. Plant Molecular Biology 66, 361-378.

Seki M., Kamei A., Yamaguchi-Shinozaki K. \& Shinozaki K. (2003) Molecular responses to drought, salinity and frost: Common and different paths for plant protection. Current Opinion Biotechnology 14, 194-199.

Seki M., Narusaka M., Ishida J., Nanjo T., Fujita M., Oono Y., Kamiya A., Nakajima M., Enju A., Sakurai T., Satou M., Akiyama K., Taji T., Yamaguchi-Shinozaki K., Carninci P., Kawai J., Hayashizaki Y. \& Shinozaki K. (2002) Monitoring the expression profiles of 7000 Arabidopsis genes under drought, cold and highsalinity stresses using a full-length cDNA microarray. Plant Journal 31, 279-292.

Seki M., Umezawa T., Urano K. \& Shinozaki K. (2007) Regulatory metabolic networks in drought stress responses. Current Opinion Plant Biology 10, 296-302.

Shelp B.J., Bown,A.W. \& McLean M.D. (1999) Metabolism and functions of gammaaminobutyric acid. Trends Plant Science 4, 446-452.

Shelp B.J., Mullen R.T. \& Waller J.C. (2012) Compartmentation of GABA metabolism raises intriguing questions. Trends Plant Science 17, 57-59.

Shi, H. \& Chan Z. (2014) The cysteine2/histidine2-type transcription factor ZINC FINGER OF ARABIDOPSIS THALIANA 6-activated C-REPEAT-BINDING FACTOR pathway is essential for melatonin-mediated freezing stress resistance in Arabidopsis. Journal of Pineal Research 57, 185-191.

Shinozaki K. \& Yamaguchi-Shinozaki K. (2007) Gene networks involved in drought stress response and tolerance. Journal of Experimental Botany 58, 221-227. 
Shinozaki K. \& Yamaguchi-Shinozaki K. (2004). Plant response to stress: regulation of plant gene expression to drought. In Encyclopedia of Plant and Crop Science. Marcel Dekker, pp 999-1001. New York.

Shinozaki K., Yamaguchi-Shinozaki K. \& Seki M. (2003) Regulatory network of gene expression in the drought and cold stress responses. Current Opinion Plant Biology 6, 410-417.

Skirycz A. \& Inzé D. (2010) More from less: plant growth under limited water. Current Opinion Biotechnology 2, 197-203.

Snedden W.A., Arazi T., Fromm H., \& Shelp B.J. (1995) Calcium/Calmodulin Activation of Soybean Glutamate Decarboxylase. Plant Physiology 108, 543-549.

Stitt M. \& Krapp A. (1999) The interaction between elevated carbon dioxide and nitrogen nutrition: the physiological and molecular background. Plant Cell Environment 22, 583-621.

Studart-Guimarães C., Fait A., Nunes-Nesi A., Carrari F., Usadel B. \& Fernie A.R. (2007) Reduced Expression of Succinyl-Coenzyme a ligase can be compensated for by up-regulation of the g-Aminobutyrate Shunt in illuminated Tomato Leaves. Plant Physiology 145, 626-639.

Supek F., Bošnjak M., Škunca N. \& Šmuc T. (2011) REVIGO summarizes and visualizes long lists of gene ontology terms. PLOS ONE 6, e21800.

Suzuki M., Kao C.Y., Cocciolone S. \& McCarty D.R. (2001) Maize VP1 complements Arabidopsis abi3 and confers a novel ABA/auxin interaction in roots. Plant J. 28, 409-418. 
Taji T., Ohsumi C., luchi S., Seki M., Kasuga M., Kobayashi M., YamaguchiShinozaki K. \& Shinozaki K. (2002) Important roles of drought and cold-inducible genes for galactinol synthase in stress tolerance in Arabidopsis thaliana. Plant Journal 29, 417-426.

Thomashow M.F. (2010) Molecular basis of plant cold acclimation: insights gained from studying the CBF cold response pathway. Plant Physiology 154, 571-577.

Toufighi K., Brady S.M. Austin R., L, E. \& Provart N.J. (2005) The botany array resource: e-Northerns, expression angling, and promoter analyses. Plant Journal 43, 153-163.

Vogel J.T., Zarka D.G., Van Buskirk H.A., Fowler S.G. \& Thomashow M.F. (2005) Roles of the CBF2 and ZAT12 transcription factors in configuring the low temperature transcriptome of Arabidopsis. Plant Journal 41, 195-211.

Wang W., Vinocur B., Shoseyov O. \& Altman A. (2004) Role of plant heat-shock proteins and molecular chaperones in the abiotic stress response. Trends Plant Science 9, 244-252.

Xiong L., Schumaker K.S. \& Zhu J.K. (2002) Cell signaling during cold, drought, and salt stress. Plant Cell 14, 165-183.

Yamaguchi-Shinozaki K. \& Shinozaki K. (2006) Transcriptional regulatory networks in cellular responses and tolerance to dehydration and cold stresses. Annual Review Plant Biology 57, 781-803. 
Yanagisawa S. (2001) The transcriptional activation domain of the plant-specific Dof1 factor functions in plant, animal, and yeast cells. Plant Cell Physiology 42 , 813-822.

Yanagisawa S. (2002) The Dof family of plant transcription factors. Trends Plant Science 7, 555-560.

Yanagisawa S., Akiyama A., Kisaka H., Uchimiya H. \& Miwa T. (2004) Metabolic engineering with Dof1 transcription factor in plants: Improved nitrogen assimilation and growth under low-nitrogen conditions. Proc. Natl. Acad. Sci. USA. 101, 78337838.

Yanagisawa S. \& Schmidt RJ. (1999) Diversity and similarity among recognition sequences of Dof transcription factors. Plant Journal 17, 209-214.

Yanagisawa S. \& Sheen J. (1998) Involvement of maize Dof zinc finger proteins in tissue-specific and light-regulated gene expression. Plant Cell 10, 75-89.

Yang X. Srivastava R. Howell SH. Bassham DC. (2016) Activation of autophagy by unfolded proteins during endoplasmic reticulum stress. Plant Journal 85, 83-95.

Yoo S.D., Cho Y.H. and Sheen J. (2007) Arabidopsis mesophyll protoplasts: a versatile cell system for transient gene expression analysis. Nature Protocols 2, 1565-1572.

Zhu J.K. (2002) Salt and drought stress signal transduction in plants. Annual Review Plant Biology 53, 247-273. 


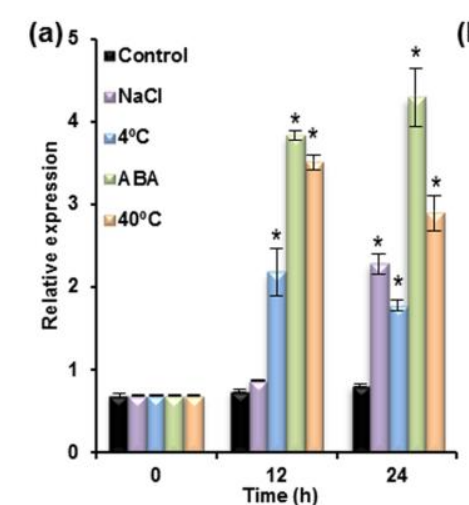

(b)

(c)

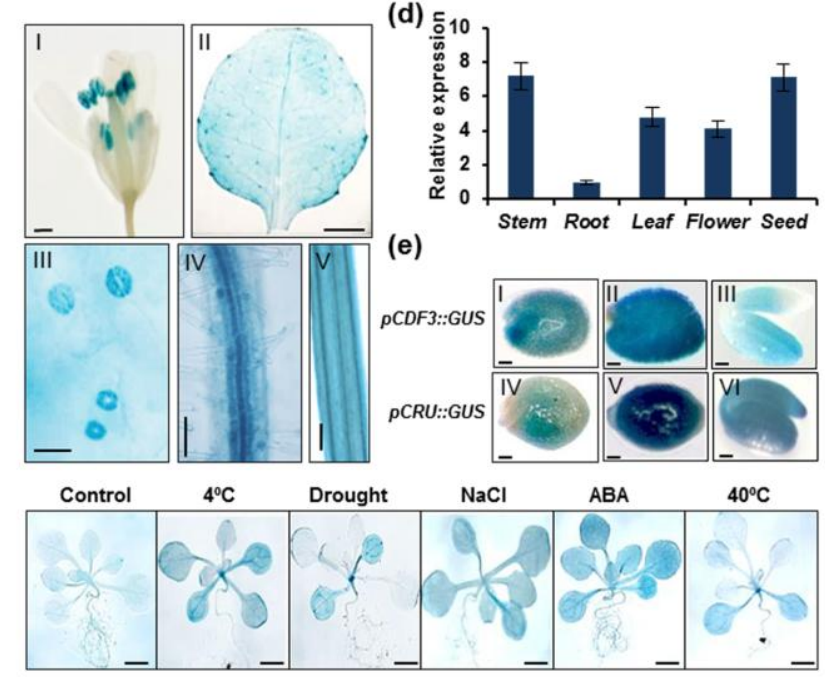

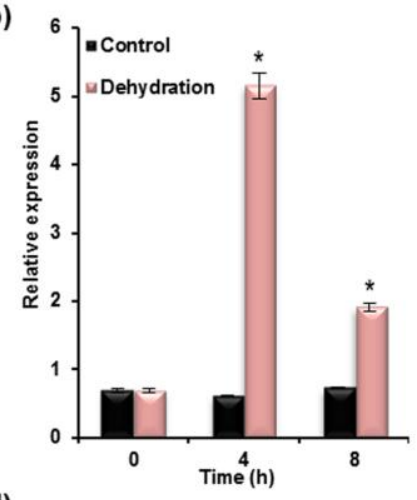

(f)

Figure 1. Expression pattern of $C D F 3$ gene in response to different abiotic stress conditions.

Figure. 1. Expression pattern of $C D F 3$ gene in response to different abiotic stress conditions.

(a-b) qRT-PCR analysis of CDF3 gene expression. Total RNA was isolated from leaves of 3-week-old Arabidopsis plants grown under control conditions (control), treated with $(A) 150 \mathrm{mM} \mathrm{NaCl}(\mathrm{NaCl})$, low temperatures $\left(4^{\circ} \mathrm{C}\right), 100 \mu \mathrm{M}$ ABA $(\mathrm{ABA})$, heat $\left(40^{\circ} \mathrm{C}\right)$ or $(\mathrm{B})$ dried on the bench (dehydration) for the indicated periods of time. Arabidopsis UBIQUITIN21 gene was used as a reference gene. Data are means \pm SE $(n=3)$. Asterisks indicate significant differences from control; * $P<0.05$; by Student's t-test. 
(c) GUS staining of $p C D F 3:: G U S$ plants showing CDF3 localization in flower and pollen I, young leaf II, stomata III, root IV and stem V. Scale bars: (I) $200 \mu \mathrm{m}$; (II) $1 \mathrm{~mm}$; (III) $80 \mu \mathrm{m} ;($ IV-V) $1 \mathrm{~cm}$.

(d) Expression pattern of CDF3 gene in different organs of adult Arabidopsis plants. qRT-PCR analyses were performed with total RNA extracted from stems, roots, leafs, flowers and seeds of 8-week-old Arabidopsis plants.

(e) GUS staining pCDF3::GUS (I-III) and pCRU::GUS (IV-VI) showing CDF3 and CRUCIFERIN expression in seeds. (I, IV) early maturation, (II, V) late maturation, (III, VI) dry seeds. Scale bars: $200 \mu \mathrm{m}$.

(f) GUS staining showing CDF3 localization in three-week-old transgenic pCDF3::GUS Arabidopsis plants grown under control conditions (control) or exposed to low $\left(4^{\circ} \mathrm{C}\right)$ or high temperature $\left(40^{\circ} \mathrm{C}\right)$, dried on the bench (drought), treated with $100 \mu \mathrm{M} A B A(A B A)$ or $150 \mathrm{mM} \mathrm{NaCl}(\mathrm{NaCl})$ for $24 \mathrm{~h}$. Scale bars: $3 \mathrm{~mm}$. 


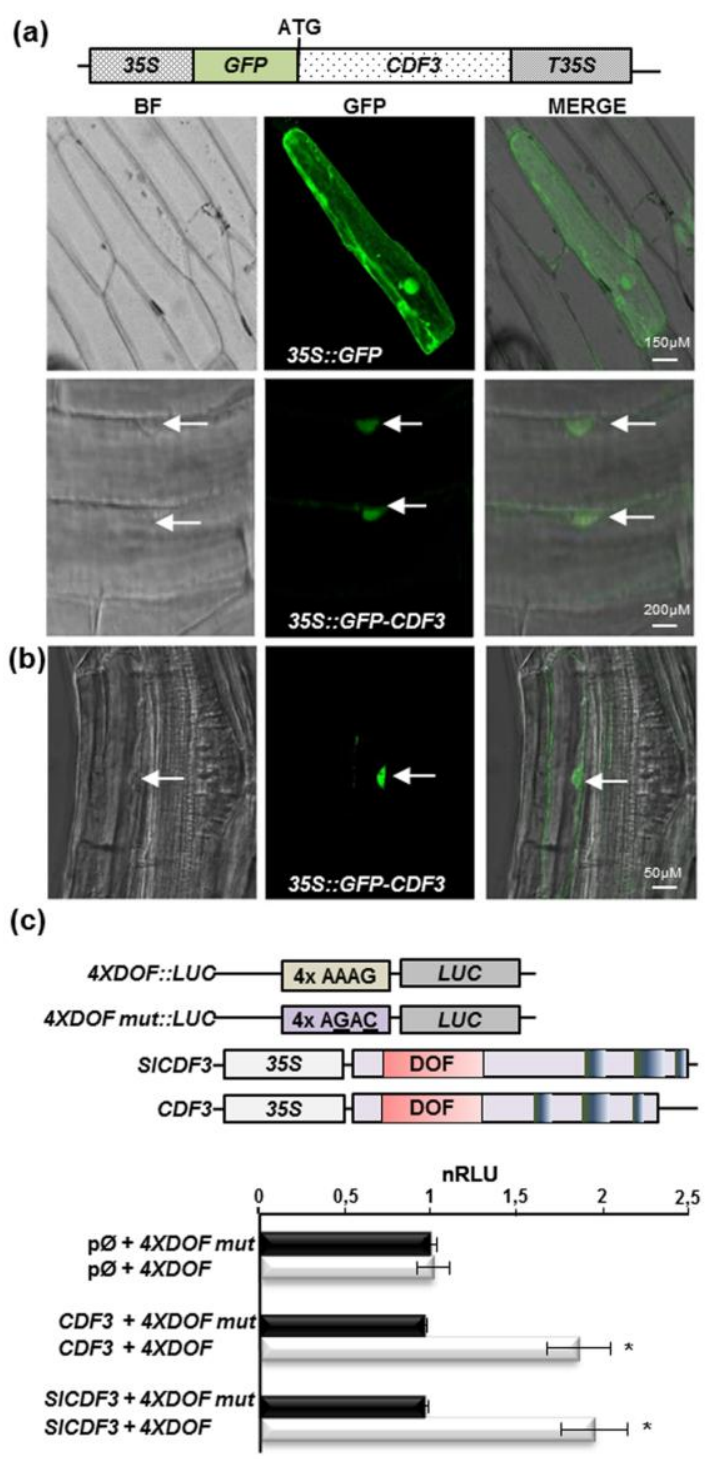

Figure 2. Subcellular localization and transcriptional activation properties of CDF3 protein.

Figure 2. Subcellular localization and transcriptional activation properties of CDF3.

(a-b) CDF3 protein is targeted to the nuclei. Onion epidermis cells (a) and Arabidopsis cells (b) were transiently and stable transformed by particle bombardment and Agrobacterium with 35S::GFP-CDF3 construct, respectively. As controls, onion layers were transformed with the 35S::GFP. Confocal images of onion and Arabidopsis root cells showing CDF3 nuclear localization (White Arrows), with an overlay of the bright field (BF) and GFP images (GFP). 
(c) CDF3 binding to the DOF motif. Transient expression assays in Arabidopsis protoplasts using 35S::CDF3 effector plasmid and the reporter plasmids 4xDOF::LUC and 4XDOFmut::LUC. Tomato homologous gene SICDF3 and empty effector plasmid $(p \phi)$ were used as positive and negative controls, respectively. Data are means $\pm \operatorname{SE}(n=3)$. Data are means $\pm S E(n=3)$. Asterisks indicate significant differences from control $(p \phi) ;{ }^{*} P<0.05$; by Student's t-test.

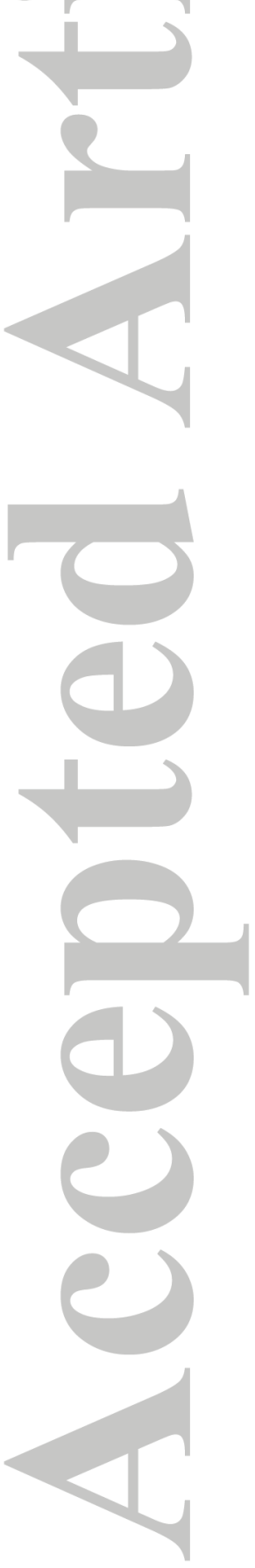




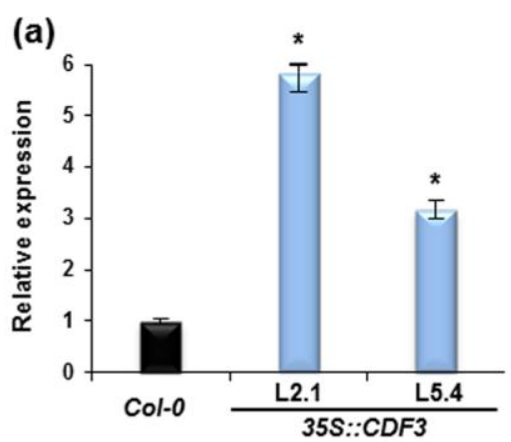

(b)

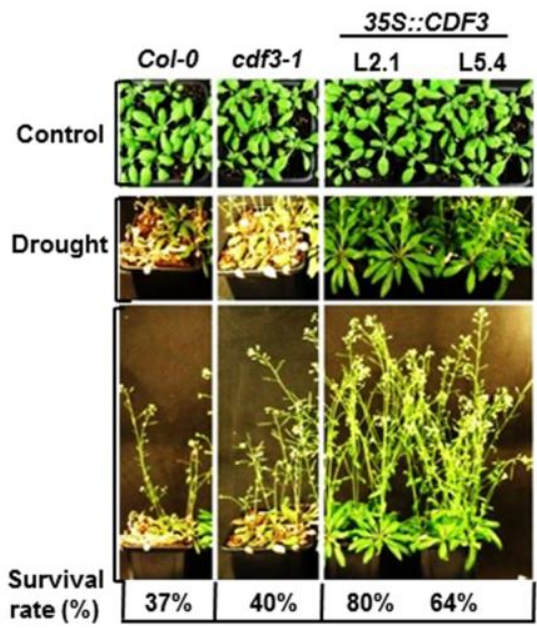

(c)

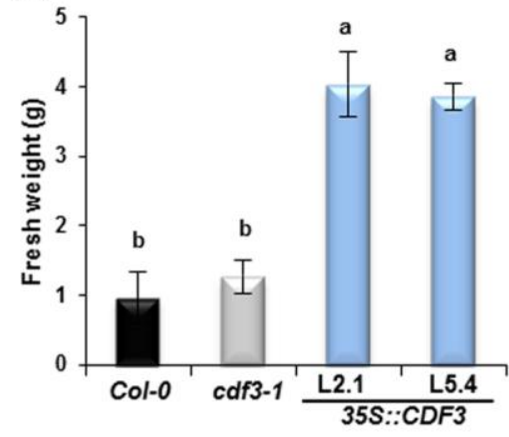

(d)

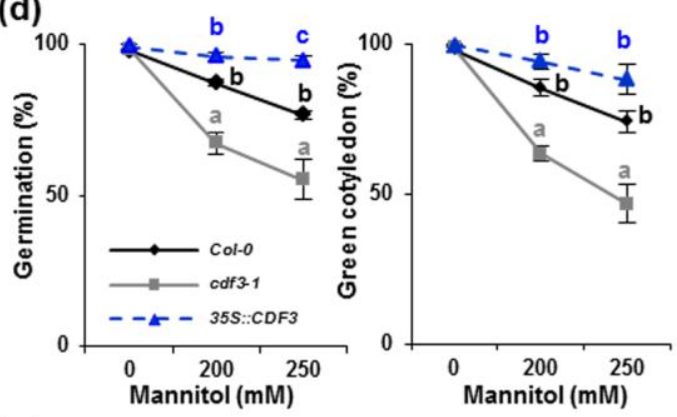

(e)

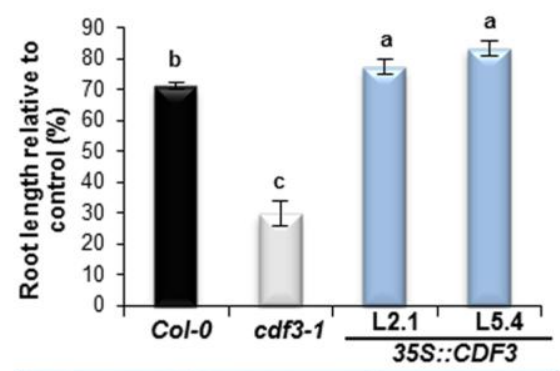

(f)

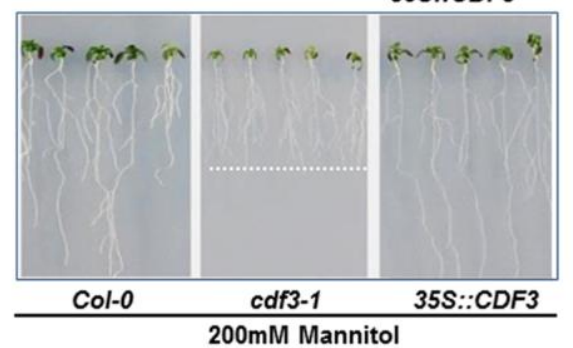

(g)

(h)
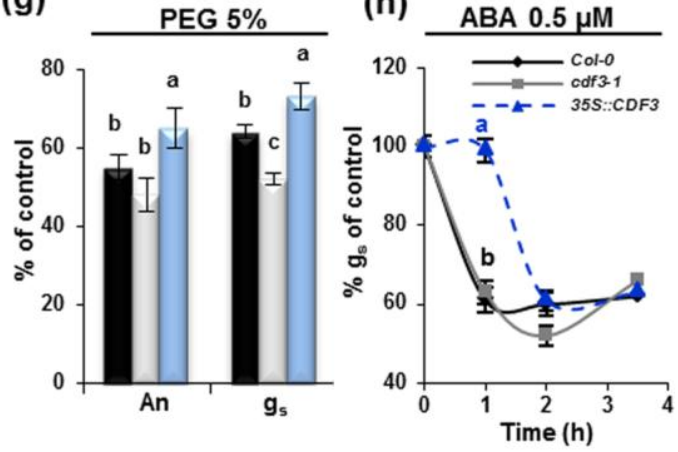

Figure 3. Effects of CDF3 on tolerance to drought and osmotic stress.

Figure 3. Effects of CDF3 on tolerance to drought and osmotic stress.

(a) qRT-PCR analysis of CDF3 expression in 35S::CDF3 (L2.1 and L5.4) transgenic lines. Data are means \pm SE $(n=3)$. Asterisks indicate significant differences from Col$0 ;{ }^{*} \mathrm{P}<0.05$ by Student's t-test. 
(b) Phenotypes and survival rates of Col-0, mutant and overexpressor plants grown under normal and dehydration conditions. The photographs and survival rates were obtained after re-watering for 10 days after dehydration treatment.

(c) Fresh weight of Col-0, cdf3-1 and 35S::CDF3 plants after dehydration treatment. Values are means \pm SE $(n=3)$. Letters indicate significant differences between Col-0, mutant and overexpresor plants; $\mathrm{P}<0.05$; ANOVA Student-Newman-Keuls tests.

(d) Germination rates and appearance of green cotyledons of 35S::CDF3, Col-0 and cdf3-1 plants that were germinated under different concentrations of mannitol. Data are means \pm SE $(n=3)$. Letters indicate significant differences between Col-0, cdf3-1 and 35S::CDF3; $\mathrm{P}<0.05$; ANOVA Student-Newman-Keuls tests.

(e-f) Root elongation assays. Six-day-old seedlings were transferred MS agar plates or supplemented with $200 \mathrm{mM}$ mannitol and incubated vertically for $10 \mathrm{~d}$ before primary root length were estimated. (e) Results are represented as percentage of reduction relative to standard conditions. Data are means \pm SE of three independent experiments with at least 20 plants each. Letters indicate significant differences between Col-0, cdf3-1 and 35S::CDF3 ( $\mathrm{P}<0.05$; ANOVA Student-Newman-Keuls tests). (f) Representative images of Col-0, cdf3-1 and 35S::CDF3 plants after treatments.

(g) Photosynthetic rate $(A n)$ and stomatal conductance $\left(g_{s}\right)$ were estimated in 3week-old Col-0, cdf3-1 and 35S::CDF3 Arabidopsis plants growth under control conditions, or treated with 5\% PEG, for $24 \mathrm{~h}$. Data were referred to the values in control conditions. Data are means \pm SE $(n=8)$. Letters indicate significant differences between Col-0, cdf3-1 and 35S::CDF3; $\mathrm{P}<0.05 ;$ ANOVA StudentNewman-Keuls tests. 
(h) The effect of $A B A$ on the reduction of stomatal conductance (gs) was estimated in four-week-old plants grown in soil by spraying with $0.5 \mu \mathrm{M}$ ABA solution in the underside of the leaves and measurements were made after 1, 2 and 3.5 hours after treatment. Date are referred to the parameter at $t=0$. Data are means $\pm S E(n=6)$. Asterisks indicate significant differences from Col-0; ${ }^{*} \mathrm{P}<0.05$, by Student's $\mathrm{t}$-test.

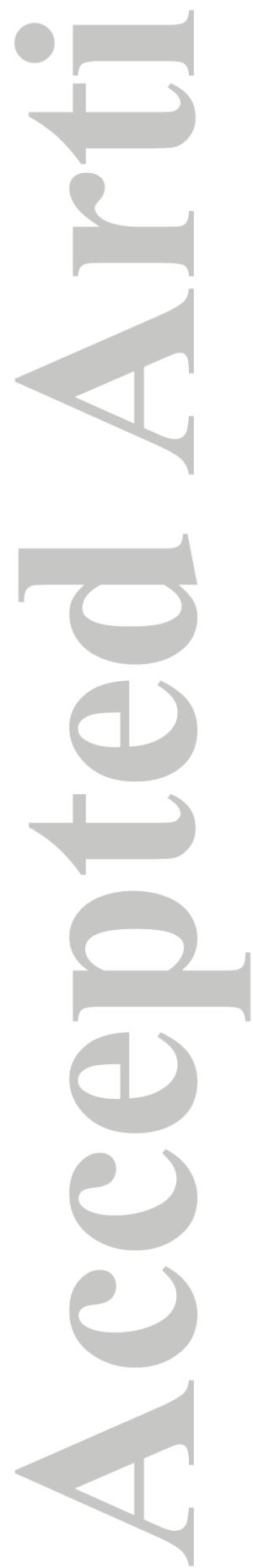


(a)

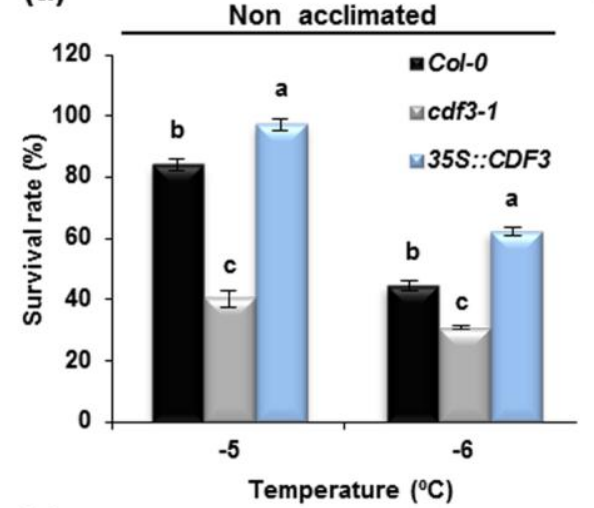

(c)

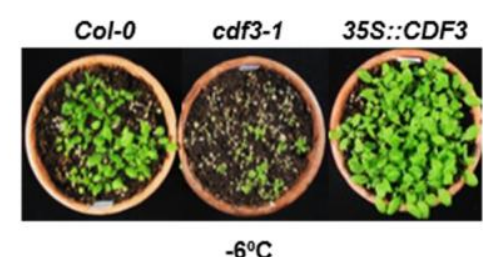

(b)

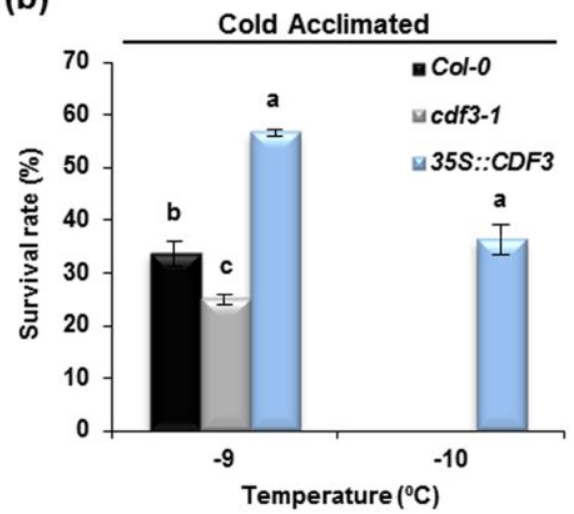

(d)

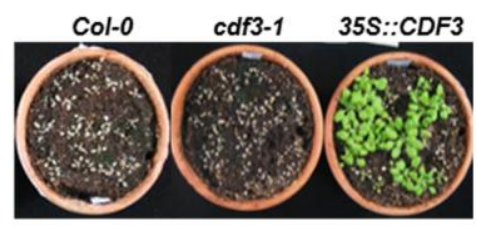

$-10^{\circ} \mathrm{C}$

Figure 4. Effects of CDF3 on tolerance to low temperatures.

Figure. 4. Effects of CDF3 on tolerance of low temperatures.

(a-b) Freezing tolerance of nonacclimated (a) and cold acclimated (7 days at $\left.4^{\circ} \mathrm{C}\right)(\mathrm{b})$ 2-week-old Col-0, 35S::CDF3 and cdf3-1 plants that were exposed to the indicated freezing temperatures for $6 \mathrm{~h}$. Freezing tolerance was estimated as the percentage of plants surviving each specific temperature after $7 d$ of recovery under control conditions. Data are expressed as means \pm SE of the three independent experiments with 50 plants each. Letters indicate significant differences between Col-0, cdf3-1 and 35S::CDF3; $\mathrm{P}<0.05$ ANOVA followed by Student-Newman-Keuls test). 
(c-d) Phenotypes of nonacclimated (c) and cold acclimated (d) Col-0, mutant and overexpressor plants after $7 \mathrm{~d}$ of recovery after being exposed to the indicated freezing temperatures.

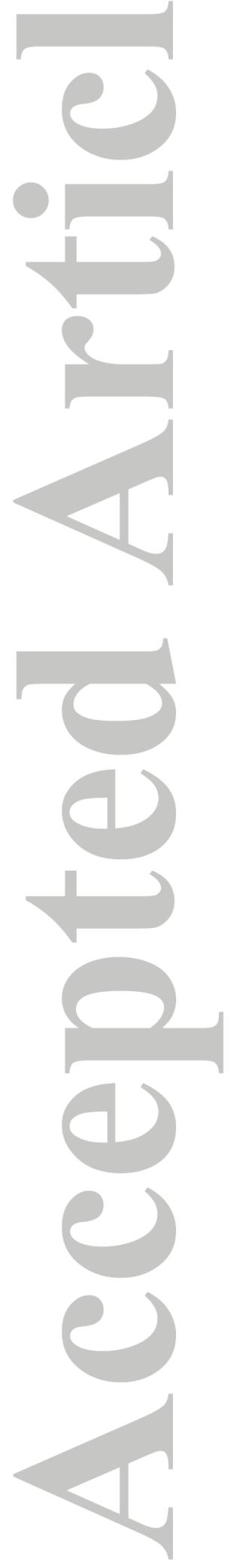


(a)

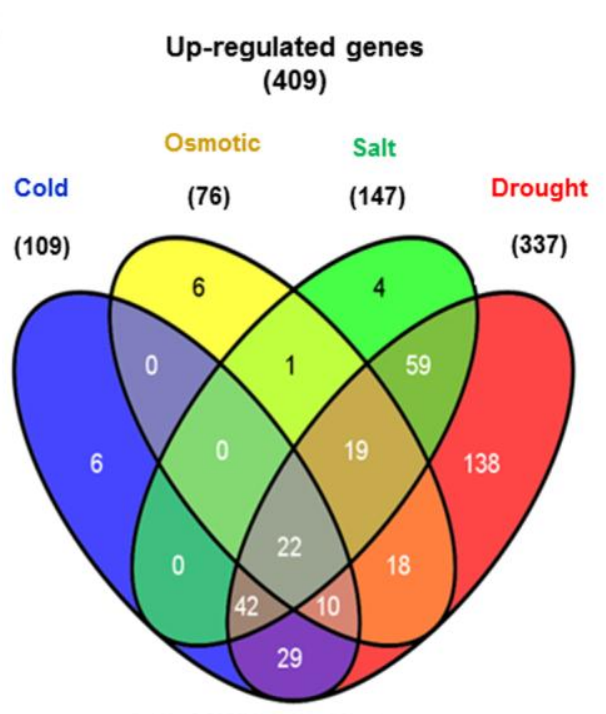

35S::CDF3_VS_WT

(c)

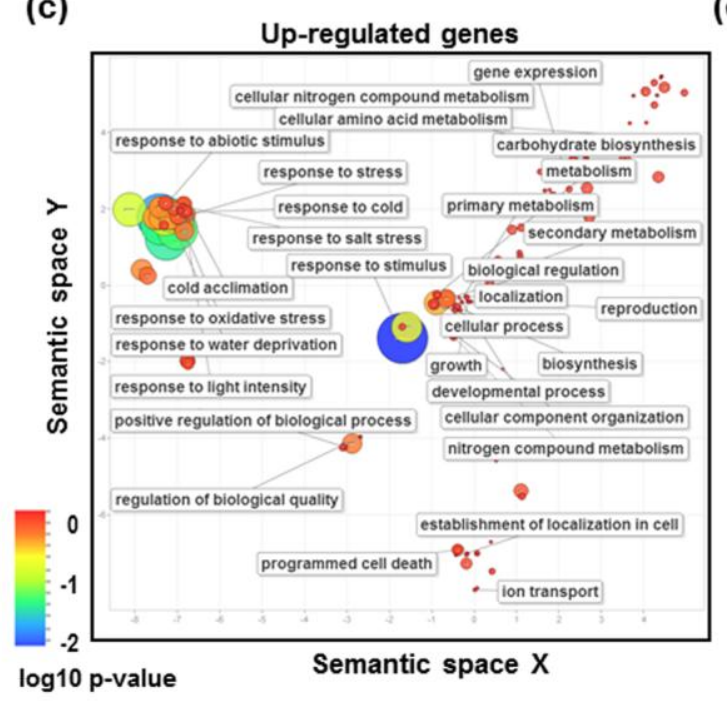

(b)

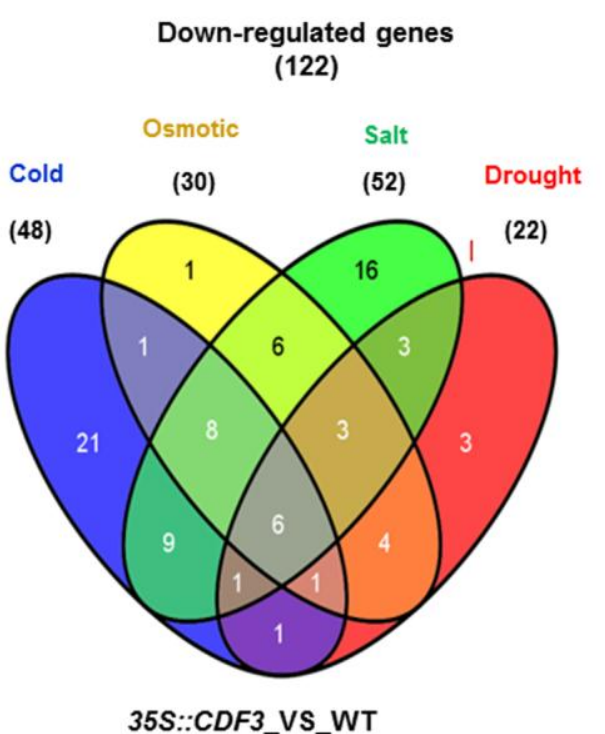

(d)

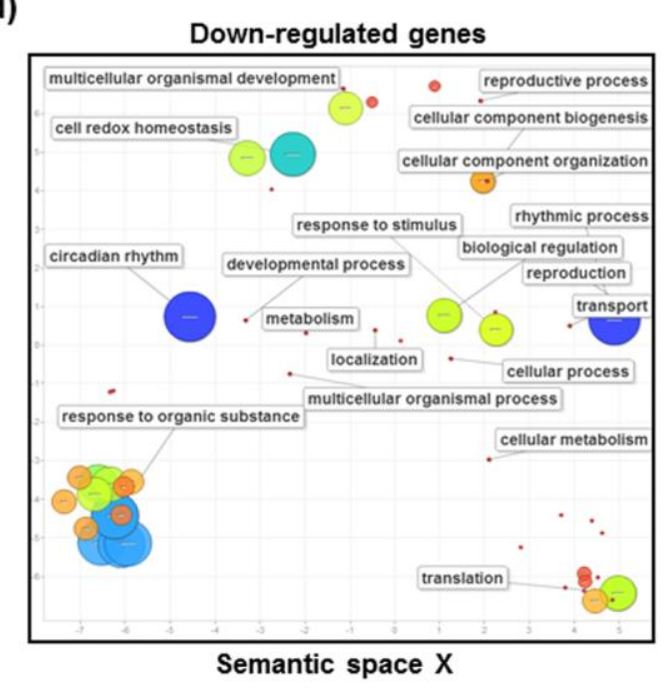

Figure. 5. Classification and Gene Ontology analyses of the genes differentially expressed in CDF3 overexpressing lines compared with WT plants.

Figure 5. Classification and Gene Ontology analyses of the genes differentially expressed in CDF3 overexpressing lines compared with WT plants.

(a-b) Venn diagrams showing overlap of (a) up-regulated and (b) down-regulated genes expressed in 35S::CDF3 transgenic plants compared with WT plants in response to different stresses. In silico expression analyses and classification of 
35S::CDF3 up-regulated and down regulated genes in response to cold, osmotic, salt and drought stresses, by using e-Northern Expression Browser tool.

(c-d) Scatter plot of (c) up-regulated and (d) down-regulated genes expressed in 35S::CDF3 compared with WT plants shows the cluster representatives (terms remaining after reducing redundancy) in a two-dimensional space. The scatter plots were performed using AgriGO and Revigo tools. Bubble color indicates the p-value for the false discovery rates derived from the AgriGO analysis as well as biological processes.

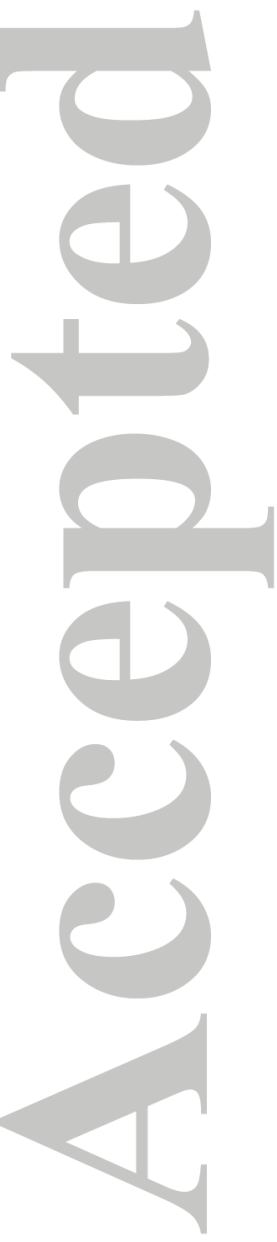



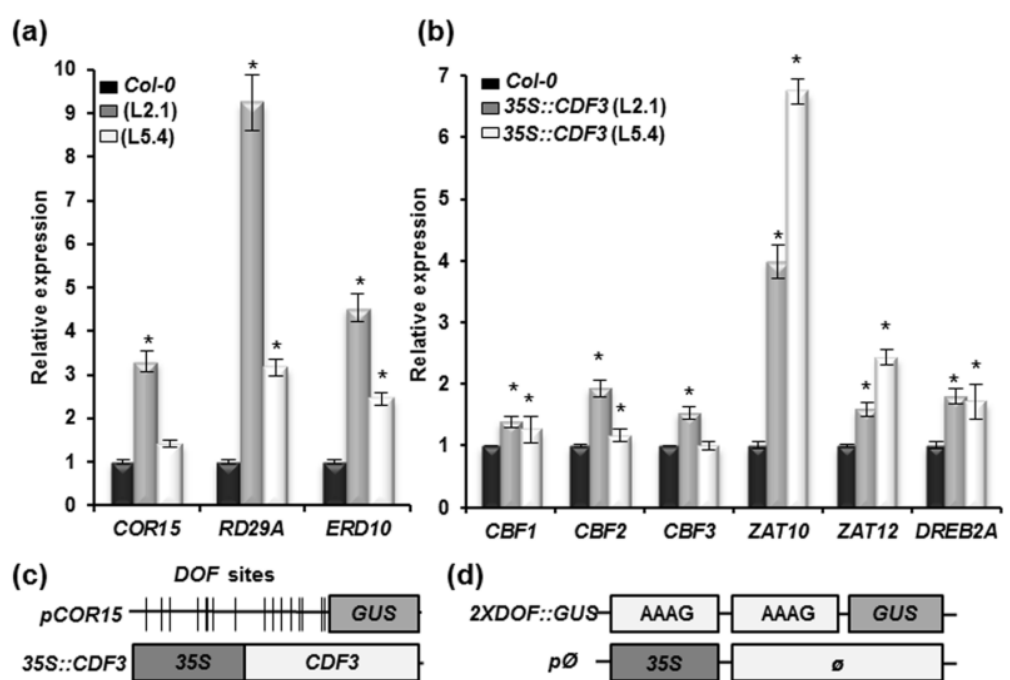

(d)
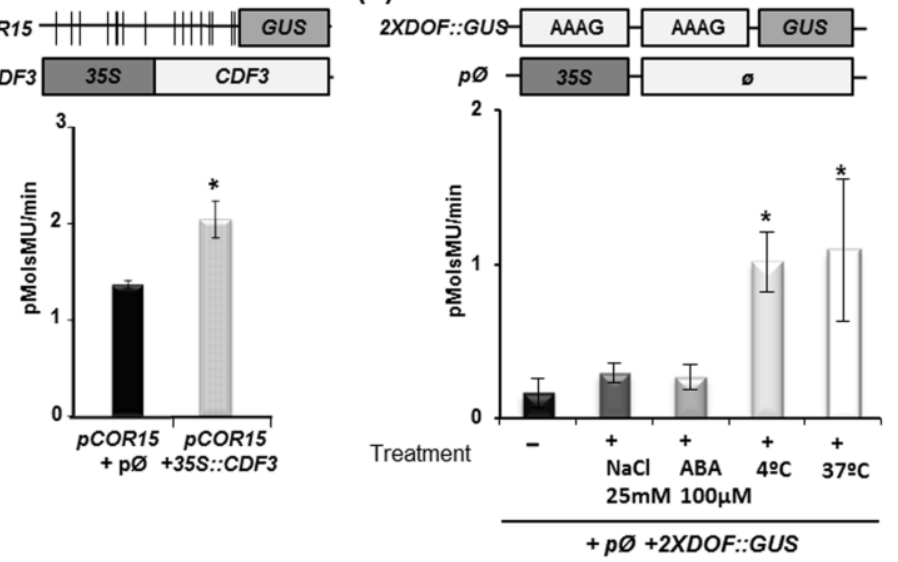

Figure 6. CDF3 regulates a set of genes involved in cellular osmoprotection and stressrelated transcription factors.

Figure 6. CDF3 regulates a set of genes involved in cellular osmoprotection and stress-related transcription factors.

(a-b) Transcription analysis by qRT-PCR of COR15, RD29A and ERD10 stressresponsive genes (a) and CBF1,CBF2, CBF3, ZAT10, ZAT12 and DREB2A genes (b) in 4-week-old 35S::CDF3 (L2.1 and L5.4) and Col-0 plants. UBIQUITIN21 gene was used as a reference gene. Data are means of $\pm \operatorname{SE}(n=5)$. Asterisks indicate significant differences from WT; * $\mathrm{P}<0.05$ by Student's t-test.

(c) Transcriptional activation assay of COR15 gene promoter by CDF3. Arabidopsis protoplasts were co-transfected with pCOR15::GUS reporter plasmid and effector 35S::CDF3 constructs. Empty effector plasmid was used as negative control. Data 
are means $\pm \operatorname{SE}(n=5)$. Asterisks indicate significant differences from control; * $\mathrm{P}<0.05$ by Student's t-test.

(d) DOF DNA motif is an abiotic stress-responsive element. Protoplasts were cotransfected with $p B T 10-2 x D O F-G U S$ reporter plasmid and empty effector plasmid and exposed to control conditions (Control) or treated with $\mathrm{NaCl}(25 \mathrm{mM})$ and $\mathrm{ABA}$ $(100 \mu \mathrm{M})$, or incubated at extreme temperatures $\left(4^{\circ} \mathrm{C}\right.$ and $\left.37^{\circ} \mathrm{C}\right)$ for $12 \mathrm{~h}$. Data are means \pm SE $(n=3)$. Asterisks indicate significant differences from Control; ${ }^{*} P<0.05$ by Student's t-test.

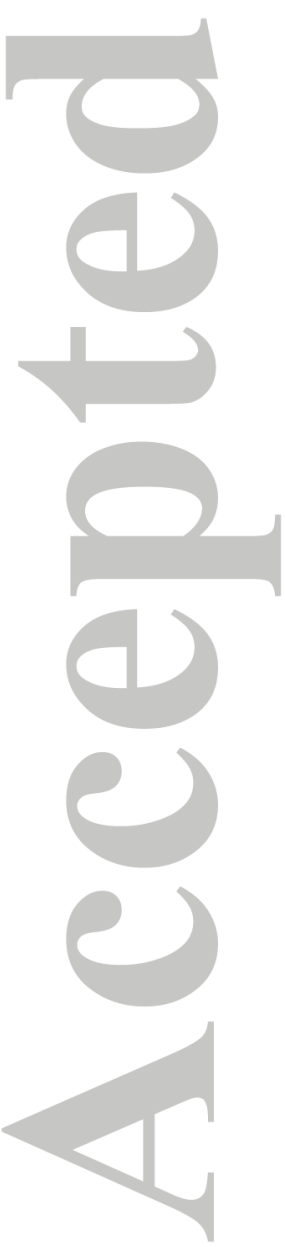



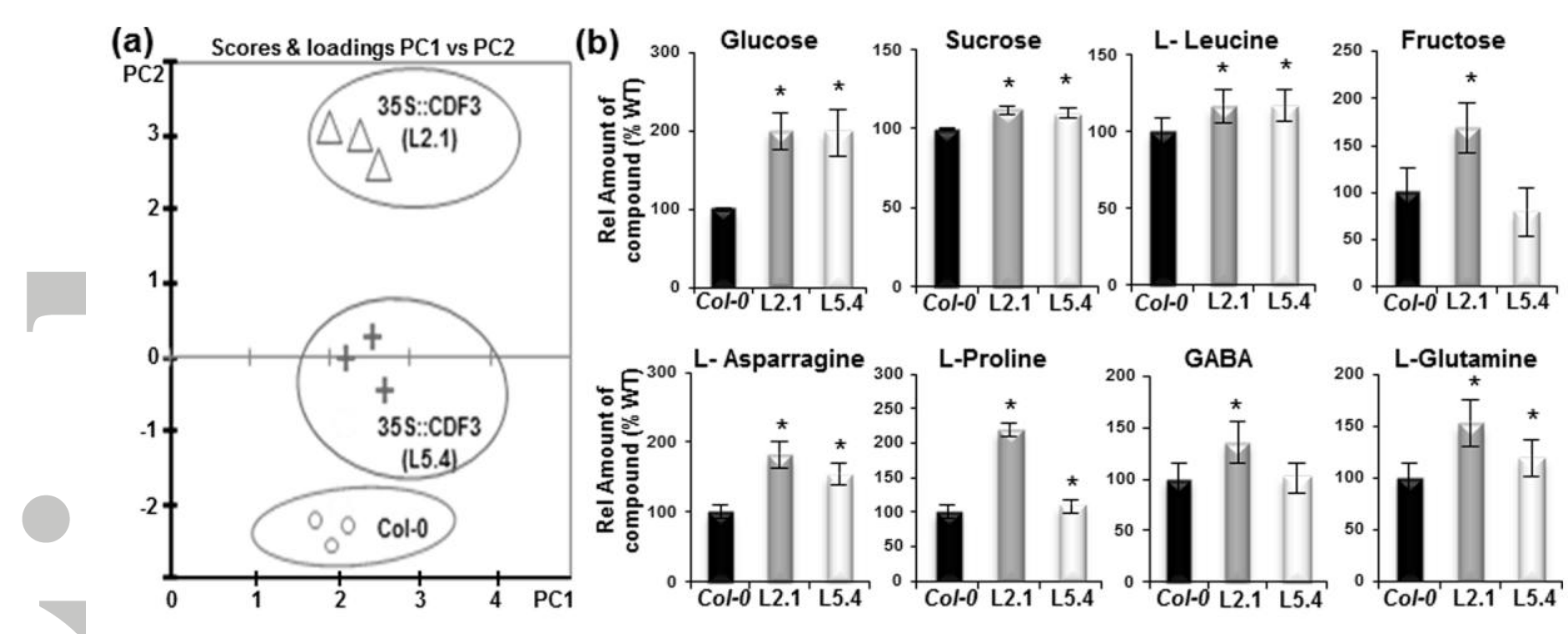

Figure 7. Effect of $C D F 3$ in sugar and amino acid metabolism.

Figure 7. Effect of CDF3 in sugar and amino acid metabolism.

(a) PCAs of recorded, non-targeted metabolic profiles. Projection plots are shown for principal component 1 (PC1, 28\% variance explained) and PC2 (55.3\%). Distinct grouping supports the different genotypes analyzed: Col-0 or overexpression lines 2.1 and 5.4 , respectively.

(b) Relative quantities (\% of WT) of selected metabolites analyzed by Gas chromatography-selected ion monitoring-mass spectrometry. Results are shown as means $\pm S E(n=15)$. Similar results were obtained in five independent experiments; * $\mathrm{P}<0.01 ;$ ANOVA, followed by a Student-Newman Keuls test. 